TRANSACTIONS OF THE

AMERICAN MATHEMATICAL SOCIETY

Volume 362, Number 4, April 2010, Pages 1869-1906

S 0002-9947(09)04756-4

Article electronically published on October 30, 2009

\title{
DEGENERATE SOBOLEV SPACES AND REGULARITY OF SUBELLIPTIC EQUATIONS
}

\author{
ERIC T. SAWYER AND RICHARD L. WHEEDEN
}

\begin{abstract}
We develop a notion of degenerate Sobolev spaces naturally associated with nonnegative quadratic forms that arise from a large class of linear subelliptic equations with rough coefficients. These Sobolev spaces allow us to make the widest possible definition of a weak solution that leads to local Hölder continuity of solutions, extending our results in an earlier work, where we studied regularity of classical weak solutions. In cases when the quadratic forms arise from collections of rough vector fields, we study containment relations between the degenerate Sobolev spaces and the corresponding spaces defined in terms of weak derivatives relative to the vector fields.
\end{abstract}

\section{INTRODUCTION}

In a recent paper [11, the authors proved both a rough analogue of the FeffermanPhong regularity theorem for smooth subelliptic self-adjoint operators, and a rough analogue of a special diagonal case of Hörmander's theorem for sums of squares of smooth vector fields. For convenience, both of these rough theorems were stated using the classical notion of weak solution defined in terms of the Sobolev space

$$
H^{1,2}(\Omega)=\left\{f \in L^{2}(\Omega): \nabla f \in L^{2}(\Omega)\right\}, \quad \Omega \text { open } \subset \mathbb{R}^{n},
$$

where $\nabla f$ is here taken in the weak or distribution sense. To illustrate briefly in the simplest of cases, a function $u \in H^{1,2}(\Omega)$ was said to be a weak solution of

$$
L u=f \text { in } \Omega \text {, }
$$

where $L=\nabla^{\prime} Q(x) \nabla$ and $f \in L^{2}(\Omega)$, provided

$$
-\int_{\Omega} \nabla v(x)^{\prime} Q(x) \nabla u(x) d x=\int_{\Omega} v(x) f(x) d x
$$

for all $v \in \operatorname{Lip}_{c}(\Omega)$, the space of Lipschitz functions with compact closure in $\Omega$. Under additional hypotheses, it was concluded in [11] that after redefinition on a set of measure zero, the weak solution $u$ was Hölder continuous in $\Omega$ of some positive order. Using this classical notion of weak solution, applications were then given in [7, 8], 9] and [1] to the Monge-Ampère equation via the partial Legendre transform.

In this paper we give the widest possible definition of a weak solution that still results in the Hölder regularity conclusion in [11 for these rough theorems. Due to the technical nature of the hypotheses and conclusions, we defer the rigorous statements of the theorems (see Theorems 16, 17 and 18 below) until we have first

Received by the editors September 6, 2007.

2000 Mathematics Subject Classification. Primary 35B65, 35D10, 35H20, 46E35.

(C)2009 American Mathematical Society Reverts to public domain 28 years from publication 
developed the requisite theory of degenerate Sobolev spaces. We begin with some heuristics. We would like to define a larger Sobolev space than $H^{1,2}(\Omega)$ for which the integrals in (2) make sense (exploiting the fact that $Q(x)$ may degenerate), but for which the calculus necessary for the proof of regularity continues to hold. One important feature in the classical case is that Lipschitz, or even smooth, functions are dense in $H^{1,2}(\Omega)$, and this density permits the transfer of the required calculus to $H^{1,2}(\Omega)$. There are thus two natural approaches in the literature to defining a notion of degenerate Sobolev space. One is denoted $H_{\mathcal{X}}^{1,2}$, where $\mathcal{X}$ is a collection of vector fields, and uses weak derivatives defined via integration by parts, in which a calculus is problematic, and the other is denoted $W_{\mathcal{Q}}^{1,2}$, where $\mathcal{Q}$ is a general quadratic form, and uses strong derivatives defined by taking strong limits of Lipschitz functions, which inherits a calculus by continuity. Before recalling precise definitions, we discuss in detail the relative merits of these two approaches.

Note that the definitions of $W$ and $H$ spaces given here and in [11] are reversed from those given elsewhere in the literature, in particular in [3] and [4. The degenerate Sobolev space $H_{\mathcal{X}}^{1,2}$ defined using weak derivatives has at least two advantages over the degenerate Sobolev space $W_{\mathcal{Q}}^{1,2}$ defined using strong derivatives:

- membership in $H_{\mathcal{X}}^{1,2}$ is easily decided using the definition of weak derivative, while membership in $W_{\mathcal{Q}}^{1,2}$ is difficult to decide using Cauchy sequences,

- the natural bounded map from $H_{\mathcal{X}}^{1,2}$ to $L^{2}$ is one-to-one while the corresponding map from $W_{\mathcal{Q}}^{1,2}$ to $L^{2}$ may not be - i.e. derivatives in $W_{\mathcal{Q}}^{1,2}$ are not uniquely determined by the $L^{2}$ component, whereas they are in $H_{\mathcal{X}}^{1,2}$, while the space $W_{\mathcal{Q}}^{1,2}$ has at least one crucial advantage over $H_{\mathcal{X}}^{1,2}$ :

- there is a calculus available for the elements in $W_{\mathcal{Q}}^{1,2}$ that is inherited by continuity from the calculus for the dense subspace of Lipschitz functions, while such a calculus is generally problematic in $H_{\mathcal{X}}^{1,2}$.

As a result of this dichotomy between $H$ and $W$ spaces, it becomes an important question to decide when these two spaces, one defined in terms of weak derivatives and the other in terms of strong derivatives, actually coincide. As we will see, these spaces always coincide in dimension $n=1$ whenever they are both defined, and we suspect they will coincide in higher dimensions as well. However, to date it is only known that they coincide in higher dimensions for a collection of Lipschitz vector fields ( $\underline{3}$ and $[4)$. We will give two generalizations of this result below, and we will also discuss the $L^{p}$ analogues of these spaces, showing in particular that they may differ when $p>2$. Finally, in case the quadratic forms $\mathcal{Q}$ and $\mathcal{X}$ are comparable, we show below that $W_{\mathcal{Q}}^{1,2}$ is naturally embedded in $H_{\mathcal{X}}^{1,2}$ (provided $\mathcal{X}$ is such that $H_{\mathcal{X}}^{1,2}$ can be defined), and as a consequence gradients are uniquely determined in $W_{\mathcal{Q}}^{1,2}$.

Here is an outline of the paper. We first define the weak degenerate Sobolev space $H_{\mathcal{X}}^{1,2}$ associated to a collection $\mathcal{X}=\left\{X_{j}\right\}_{j=1}^{m}$ of $H_{\text {div }}^{1,2}(\Omega)$ vector fields, and show that $H_{\mathcal{X}}^{1,2}$ is a Hilbert space. We then define the strong degenerate Sobolev space $W_{\mathcal{Q}}^{1,2}$ associated to a locally integrable quadratic form $\mathcal{Q}(x, \xi)$, and give the definition of a degenerate $W_{\mathcal{Q}}$-weak solution to a subelliptic equation. In doing this we define a form-weighted Hilbert space $\mathcal{L}^{2}(\Omega, \mathcal{Q})$ in which live the (not necessarily unique) gradients of functions in $W_{\mathcal{Q}}^{1,2}$. We then give analogues for $W_{\mathcal{Q}^{-}}$weak solutions of the subellipticity theorems in [11. These analogues rely crucially on the definition 
of strong derivatives in $W_{\mathcal{Q}}^{1,2}$ spaces, as well as on the identification of gradients $\nabla w$ as belonging to $\mathcal{L}^{2}(\Omega, \mathcal{Q})$ for $w \in W_{\mathcal{Q}}^{1,2}$.

As the strong spaces $W_{\mathcal{X}}^{1,2}$ are naturally imbedded in the weak spaces $H_{\mathcal{X}}^{1,2}$ (here $\mathcal{X}$ denotes both a collection of vector fields and its associated quadratic form), and because of the dichotomy between $H$ and $W$ spaces discussed above, the question arises of when the weak and strong spaces $H_{\mathcal{X}}^{1,2}$ and $W_{\mathcal{X}}^{1,2}$ coincide. In particular these spaces are shown to always coincide in dimension $n=1$. In higher dimensions, it is known that $H_{\mathcal{X}}^{1,2}=W_{\mathcal{X}}^{1,2}$ when $\mathcal{X}$ is a collection of Lipschitz vector fields. We give two generalizations of this: when $\mathcal{X} \in H^{1,2 \sigma^{\prime}}(\Omega)$ and a Sobolev inequality holds with exponent $\sigma \geq 1$; and when $\mathcal{X} \in H^{1,2}(\Omega)$ is comparably Lipschitz off its common zero set $Z$, and Lipschitz at $Z$.

Finally, we briefly consider $H_{\mathcal{X}}^{1, p}$ and $W_{\mathcal{X}}^{1, p}$ for $\mathcal{X} \in H_{\text {div }}^{1, p^{\prime}}(\Omega)$ and $1 \leq p<$ $\infty$, in part because they are needed when taking products of elements in $H_{\mathcal{X}}^{1,2}$ or $W_{\mathcal{X}}^{1,2}$. Again, it is known that $H_{\mathcal{X}}^{1, p}=W_{\mathcal{X}}^{1, p}$ when the vector fields $\mathcal{X}$ are Lipschitz. However, we show that for $p>2$ equality may fail, and in fact when $n=1$, we show that $H_{\mathcal{X}}^{1, p}=W_{\mathcal{X}}^{1, p}$ for all $\mathcal{X} \in H_{\text {div }}^{1, p^{\prime}}(\Omega)$ if and only if $1 \leq p \leq 2$.

\section{Weak degenerate Sobolev spaces}

Define $H_{\text {div }}^{1,2}(\Omega)$ to be the linear space consisting of those $\mathbb{R}^{n}$-valued functions $\mathbf{v}$ such that

$$
\begin{aligned}
\mathbf{v} & \in L^{2}(\Omega), \\
\operatorname{div} \mathbf{v} & =\nabla \cdot \mathbf{v} \in L^{2}(\Omega),
\end{aligned}
$$

where the divergence above is taken in the sense of distributions. With the inner product

$\langle\mathbf{v}, \mathbf{w}\rangle_{H_{\text {div }}^{1,2}(\Omega)}=\int_{\Omega} \mathbf{v}(x) \cdot \mathbf{w}(x) d x+\int_{\Omega}(\nabla \cdot \mathbf{v})(x)(\nabla \cdot \mathbf{w})(x) d x, \quad \mathbf{v}, \mathbf{w} \in H_{\operatorname{div}}^{1,2}(\Omega)$,

a standard proof shows that $H_{\text {div }}^{1,2}(\Omega)$ is complete with respect to the corresponding norm, and hence $H_{\text {div }}^{1,2}(\Omega)$ is a Hilbert space. We have the following counterpart of the calculus in $H^{1,2}(\Omega)$ with $C_{c}^{1}(\Omega)$ denoting the space of continuously differentiable functions with compact support in $\Omega$ :

Lemma 1. Suppose $\mathbf{v} \in H_{\text {div }}^{1,2}(\Omega)$ and let $\left\{J_{\varepsilon}\right\}_{\varepsilon>0}$ be a smooth Euclidean approximate identity.

(1) Then $J_{\varepsilon} * \mathbf{v} \rightarrow \mathbf{v}$ locally in $H_{\text {div }}^{1,2}(\Omega)$ as $\varepsilon \rightarrow 0$.

(2) If $\varphi \in C_{c}^{1}(\Omega)$, then $\varphi \mathbf{v} \in H_{\mathrm{div}}^{1,2}(\Omega)$ and $\nabla \cdot(\varphi \mathbf{v})=\varphi \nabla \cdot \mathbf{v}+\nabla \varphi \cdot \mathbf{v}$.

Proof. We follow the analogous proofs for $H^{1,2}(\Omega)$ given in e.g. chapter 7 of [5]. If $K$ is a compact subset of $\Omega$, then both

$$
J_{\varepsilon} * \mathbf{v} \rightarrow \mathbf{v} \text { and } \nabla \cdot\left(J_{\varepsilon} * \mathbf{v}\right)=J_{\varepsilon} *(\nabla \cdot \mathbf{v}) \rightarrow \nabla \cdot \mathbf{v},
$$

in $L^{2}(K)$ as $\varepsilon \rightarrow 0^{+}$, and this proves the first assertion. The second assertion is a standard result in distribution theory if $\varphi \in C_{c}^{\infty}(\Omega)$, and to prove the case $\varphi \in C_{c}^{1}(\Omega)$, we take a sequence $\left\{\varphi_{k}\right\}_{k=1}^{\infty}$ of smooth functions with compact support converging to $\varphi$ in $C^{1}(\Omega)$, and let $k \rightarrow \infty$ in the $L^{2}(\Omega)$ identity $\nabla \cdot\left(\varphi_{k} \mathbf{v}\right)=$ $\varphi_{k} \nabla \cdot \mathbf{v}+\nabla \varphi_{k} \cdot \mathbf{v}$ 
Let $\mathcal{X}=\left\{X_{j}\right\}_{j=1}^{m}$ be a collection of $H_{\text {div }}^{1,2}(\Omega)$ vector fields on $\Omega \subset \mathbb{R}^{n}$, i.e. $X_{j}(x)=\mathbf{v}_{j}(x) \cdot \nabla$, where $\mathbf{v}_{j} \in H_{\text {div }}^{1,2}(\Omega)$ for $1 \leq j \leq m$ (in particular this includes Lipschitz vector fields when $\Omega$ is bounded). In analogy with the definition of weak partial derivative $\frac{\partial}{\partial x_{\ell}}=\mathbf{e}_{\ell} \cdot \nabla$, we say that a locally square integrable function $f$ is the weak derivative $X w$ of a locally square integrable function $w$, where $X=$ $\mathbf{v}(x) \cdot \nabla$ and $\mathbf{v}$ is in $H_{\text {div }}^{1,2}(\Omega)$, if

$$
\int_{\Omega} f \varphi=-\int_{\Omega} w X^{\prime} \varphi=-\int_{\Omega} w \nabla \cdot(\mathbf{v} \varphi)=-\int_{\Omega} w\{\varphi(\nabla \cdot \mathbf{v})+\mathbf{v} \cdot \nabla \varphi\}
$$

for all $\varphi \in C_{c}^{1}(\Omega)$. Note that the last integral converges absolutely for $\mathbf{v} \in H_{\text {div }}^{1,2}(\Omega)$. Clearly the weak derivative $X w$ is unique if it exists, and of course $X w$ exists in the weak sense, and is the $L_{l o c}^{2}(\Omega)$ function $\mathbf{v}(x) \cdot \nabla w(x)$, if $w \in \operatorname{Lip}_{l o c}(\Omega)$, the space of locally Lipschitz continuous functions on $\Omega$. Let $\mathcal{X} w=\left(X_{1} w, \ldots, X_{m} w\right)$.

We define the degenerate Sobolev space $H_{\mathcal{X}}^{1,2}(\Omega)$ as the inner product space consisting of all $w \in L^{2}(\Omega)$ whose weak derivatives $X_{j} w$ are also in $L^{2}(\Omega)$ with inner product given by

$$
\langle w, v\rangle_{\mathcal{X}}=\int_{\Omega} w \bar{v} d x+\int_{\Omega} \mathcal{X} w \cdot \overline{\mathcal{X}} v d x
$$

Note that

$$
\|w\|_{H_{\mathcal{X}}^{1,2}(\Omega)}^{2}=\|w\|_{L^{2}(\Omega)}^{2}+\|\mathcal{X} w\|_{L^{2}(\Omega)}^{2} .
$$

Theorem 2. If $\mathcal{X}$ is a collection of $H_{\mathrm{div}}^{1,2}(\Omega)$ vector fields on $\Omega$, then $H_{\mathcal{X}}^{1,2}(\Omega)$ is a Hilbert space with the inner product $\langle\cdot, \cdot\rangle_{\mathcal{X}}$ given in (4).

Proof. We prove completeness in the same way as for the classical space $H^{1,2}(\Omega)$. If $\left\{w_{k}\right\}_{k=1}^{\infty}$ is Cauchy in $H_{\mathcal{X}}^{1,2}(\Omega)$, then $\left\{w_{k}\right\}_{k=1}^{\infty}$ and $\left\{\mathcal{X} w_{k}\right\}_{k=1}^{\infty}$ are Cauchy in $L^{2}(\Omega)$ and $\bigoplus_{j=1}^{m} L^{2}(\Omega)$ respectively. Thus there are $w, f_{1}, \ldots, f_{m} \in L^{2}(\Omega)$ such that $w_{k} \rightarrow w$ and $X_{j} w_{k} \rightarrow f_{j}$ in $L^{2}(\Omega)$ for $1 \leq j \leq m$. We must now show that $f_{j}=X_{j} w$ in the weak sense. Suppose $X_{j}=\mathbf{v}_{j}(x) \cdot \nabla$. Letting $k \rightarrow \infty$ in the equation

$$
\int_{\Omega}\left(X_{j} w_{k}\right) \varphi d x=-\int_{\Omega} w_{k} \nabla \cdot\left(\mathbf{v}_{j} \varphi\right) d x, \quad \varphi \in C_{c}^{1}(\Omega),
$$

yields $\int_{\Omega} f_{j} \varphi d x=-\int_{\Omega} w \nabla \cdot\left(\mathbf{v}_{j} \varphi\right) d x$ for all $\varphi \in C_{c}^{1}(\Omega)$ as required. Thus $w \in$ $H_{\mathcal{X}}^{1,2}(\Omega)$ and we now compute that

$$
\left\|w-w_{k}\right\|_{H_{\mathcal{X}}^{1,2}(\Omega)}^{2}=\lim _{\ell \rightarrow \infty}\left\|w_{\ell}-w_{k}\right\|_{H_{\mathcal{X}}^{1,2}(\Omega)}^{2} \rightarrow 0 \text { as } k \rightarrow \infty,
$$

since $\left\{w_{k}\right\}_{k=1}^{\infty}$ is Cauchy in $H_{\mathcal{X}}^{1,2}(\Omega)$. This proves that $w_{k} \rightarrow w$ in $H_{\mathcal{X}}^{1,2}(\Omega)$.

Remark 3. We can of course define the analogous degenerate Sobolev space of $L^{p}$ functions with weak derivatives in $L^{p}$. Given $1 \leq p<\infty$ and $X=\mathbf{v}(x) \cdot \nabla$ in $H_{\text {div }}^{1, p^{\prime}}(\Omega)$, i.e. $\mathbf{v}, \operatorname{div} \mathbf{v} \in L_{l o c}^{p^{\prime}}(\Omega)$ in the sense of distributions, we say that $f \in L_{l o c}^{p}(\Omega)$ is the weak derivative $X w$ of $w \in L_{l o c}^{p}(\Omega)$ if (3) holds for all $\varphi \in C_{c}^{1}(\Omega)$. Note that all of the integrals in (3) are absolutely convergent for $f, w \in L_{l o c}^{p}(\Omega)$ and $\mathbf{v} \in H_{\operatorname{div}}^{1, p^{\prime}}(\Omega)$. Given a collection $\mathcal{X}=\left\{X_{j}\right\}_{j=1}^{m}$ of $H_{\operatorname{div}}^{1, p^{\prime}}(\Omega)$ vector fields on $\Omega \subset \mathbb{R}^{n}$, we define the degenerate Sobolev space $H_{\mathcal{X}}^{1, p}(\Omega)$ to be the linear space consisting of all $w \in L^{p}(\Omega)$ whose weak derivatives $X_{j} w$ are in $L^{p}(\Omega)$. The space 
$H_{\mathcal{X}}^{1, p}(\Omega)$ becomes a Banach space when equipped with the norm $\|w\|_{H_{\mathcal{X}}^{1, p}(\Omega)}=$ $\left\{\|w\|_{L^{p}(\Omega)}^{p}+\|\mathcal{X} w\|_{L^{p}(\Omega)}^{p}\right\}^{\frac{1}{p}}$.

As mentioned above, the calculus is in general problematic for the degenerate Sobolev space $H_{\mathcal{X}}^{1,2}(\Omega)$. Particular difficulties include the product rule and composition with a function in $C^{1}(\mathbb{R})$. Nevertheless, as we now show, multiplication by a compactly supported continuously differentiable function is well behaved on $H_{\mathcal{X}}^{1,2}(\Omega)$ and leads to the existence of a large supply of compactly supported functions in $H_{\mathcal{X}}^{1,2}(\Omega)$. If $w \in H_{\mathcal{X}}^{1,2}(\Omega)$ and $\psi \in C_{c}^{1}(\Omega)$, then $\psi w \in\left(H_{\mathcal{X}}^{1,2}\right)_{c}(\Omega)$ since $X(\psi w)=\psi X w+(X \psi) w$ in the weak sense. Indeed, writing $X=\mathbf{v} \cdot \nabla$ with $\mathbf{v} \in H_{\operatorname{div}}^{1,2}(\Omega)$ and $\varphi \in C_{c}^{1}(\Omega)$, we have the identity

$$
X^{\prime}(\varphi \psi)=\nabla \cdot(\psi \varphi \mathbf{v})=\psi \nabla \cdot(\varphi \mathbf{v})+(\varphi \mathbf{v}) \cdot \nabla \psi=\psi X^{\prime} \varphi+\varphi X \psi
$$

in $L^{2}(\Omega)$ where gradients are taken in the distribution sense. Since $C_{c}^{\infty}(\Omega)$ is dense in $L^{2}(\Omega)$, we can multiply both sides of the identity by $w \in L^{2}(\Omega)$ and integrate over $\Omega$ to obtain equality. Thus we obtain with $f=\psi X w+(X \psi) w$, and using that $X w$ is the weak derivative of $w$,

$$
\begin{aligned}
\int_{\Omega} f \varphi & =\int_{\Omega}\{\psi X w+(X \psi) w\} \varphi \\
& =-\int_{\Omega} w X^{\prime}(\varphi \psi)+\int_{\Omega} \varphi w X \psi \\
& =-\int_{\Omega} w\left\{\psi X^{\prime} \varphi+\varphi X \psi\right\}+\int_{\Omega} \varphi w X \psi \\
& =-\int_{\Omega}(\psi w) X^{\prime} \varphi
\end{aligned}
$$

for all $\varphi \in C_{c}^{1}(\Omega)$.

We emphasize that without a suitable calculus for our degenerate Sobolev space $H_{\mathcal{X}}^{1,2}(\Omega)$, we are unable to prove a regularity theorem for weak solutions based on $H_{\mathcal{X}}^{1,2}(\Omega)$. This will be rectified in the next section by introducing the degenerate Sobolev spaces with strong derivatives, and whose definition is given most naturally in the more general setting of quadratic forms rather than vector fields.

\section{Strong degenerate Sobolev spaces and $W$-weak solutions}

Given a locally integrable, nonnegative semidefinite, symmetric quadratic form $\mathcal{Q}(x, \xi)=\xi^{\prime} Q(x) \xi$ on $\Omega \subset \mathbb{R}^{n}$, i.e.

$$
\int_{L}\|Q(x)\| d x<\infty \text { for all compact } L \subset \Omega,
$$

where $\|Q\|$ is the operator norm on $n \times n$ matrices (all norms on a finite dimensional space are equivalent), we can define the form-weighted vector-valued $L^{2}$ space $\mathcal{L}^{2}(\Omega, \mathcal{Q})$ as consisting of all measurable $\mathbb{R}^{n}$-valued functions $\mathbf{f}(x)=$ $\left(f_{1}(x), \ldots, f_{n}(x)\right), x \in \Omega$, satisfying

$$
\|\mathbf{f}\|_{\mathcal{L}^{2}(\Omega, \mathcal{Q})}=\left\{\int_{\Omega} \mathcal{Q}(x, \mathbf{f}(x)) d x\right\}^{\frac{1}{2}}<\infty .
$$


If we identify measurable $\mathbb{R}^{n}$-valued functions $\mathbf{f}$ and $\mathbf{g}$ that satisfy $\|\mathbf{f}-\mathbf{g}\|_{\mathcal{L}^{2}(\Omega, \mathcal{Q})}=$ 0 , then (5) defines a norm on the resulting vector space of equivalence classes of measurable $\mathbb{R}^{n}$-valued functions. Of course $\|\mathbf{f}-\mathbf{g}\|_{\mathcal{L}^{2}(\Omega, \mathcal{Q})}=0$ if $\mathbf{f}=\mathbf{g}$ off a set of measure zero, but a characterization of when the norm vanishes requires (10) below, a representation of the norm as a sum of $L^{2}\left(\lambda_{j}\right)$ norms of components with weights $\lambda_{j}(x)$, the eigenvalues of $Q(x)$. We now suppose as usual that $\mathcal{L}^{2}(\Omega, \mathcal{Q})$ consists of these equivalence classes. Note that the representative functions in the equivalence classes are $\mathbb{R}^{n}$-valued everywhere, but we can and will consider below functions defined only almost everywhere, e.g. gradients of Lipschitz functions.

Theorem 4. The linear space $\mathcal{L}^{2}(\Omega, \mathcal{Q})$ is complete with respect to the norm (5), and is in fact a Hilbert space with respect to the associated inner product

$$
\langle\mathbf{f}, \mathbf{g}\rangle_{\mathcal{L}^{2}(\Omega, \mathcal{Q})}=\int_{\Omega} \mathbf{f}(x)^{\prime} Q(x) \mathbf{g}(x) d x .
$$

Before beginning the proof it will be helpful to express the norm (5) in terms of weighted $L^{2}$-norms of scalar functions. Let $\left\{\lambda_{j}(x)\right\}_{j=1}^{n}$ be an enumeration of the eigenvalues of $Q(x)$ arranged in decreasing order,

$$
\lambda_{j+1}(x) \leq \lambda_{j}(x) \text { for } 1 \leq j<n,
$$

and let $\left\{\mathbf{v}_{j}(x)\right\}_{j=1}^{n}$ be corresponding orthogonal unit eigenvectors.

Remark 5 . The functions $\lambda_{j}(x)$ are uniquely determined by (7) and are Lebesgue measurable since $Q(x)$ is, while the vector functions $\mathbf{v}_{j}(x)$ may be chosen to be Lebesgue measurable. To see these assertions, define the lexicographic order $\succeq$ on $\mathbb{R}^{n}$ by declaring

$$
\xi=\left(\xi_{1}, \ldots, \xi_{n}\right) \succ \eta=\left(\eta_{1}, \ldots, \eta_{n}\right)
$$

if $\xi_{k}>\eta_{k}$ where $k$ is the least index $j$ such that $\xi_{j} \neq \eta_{j}$. Note that every compact subset $E$ of $\mathbb{R}^{n}$ has a unique element that is maximal with respect to lexicographic order. Indeed, if $L_{j}(\alpha)$ is the closed half-plane $\left\{\xi \in \mathbb{R}^{n}: \xi_{j} \geq \alpha\right\}$, then the unique maximal element $\xi$ in $E$ satisfies

$$
\begin{aligned}
& \xi_{1}=\sup \left\{\eta_{1}: E \cap L_{1}\left(\eta_{1}\right) \neq \phi\right\}, \\
& \xi_{2}=\sup \left\{\eta_{2}: E \cap L_{1}\left(\xi_{1}\right) \cap L_{2}\left(\eta_{2}\right) \neq \phi\right\},
\end{aligned}
$$

where the suprema are attained by the finite intersection property of compact sets.

Now $\lambda_{1}(x)=\sup \left\{\xi^{\prime} Q(x) \xi: \xi \in \mathbb{S}^{n-1}\right\}$ is a measurable function of $x$ since we may take the supremum over a countable dense subset of the sphere $\mathbb{S}^{n-1}$. We then choose $\mathbf{v}_{1}(x)$ to be maximal with respect to lexicographic order in the compact set

$$
E_{1}(x)=\left\{\xi \in \mathbb{S}^{n-1}: \xi^{\prime} Q(x) \xi=\lambda_{1}(x)\right\}
$$

This is a measurable function of $x$ since in defining the components of the maximal element $\mathbf{v}_{1}(x)$ in (8), we may restrict the suprema to be taken over rational numbers. With $\lambda_{j}(x)$ and $\mathbf{v}_{j}(x)$ defined and measurable for $1 \leq j \leq k<n$, we have by the variational formulas for eigenvalues (easily obtained by diagonalizing matrices)

$$
\lambda_{k+1}(x)=\sup \left\{\xi^{\prime} Q(x) \xi: \xi \in \mathbb{S}^{n-1}, \xi \cdot \mathbf{v}_{j}(x)=0,1 \leq j \leq k\right\},
$$


and we choose $\mathbf{v}_{k+1}(x)$ to be maximal with respect to the lexicographic order in the compact set

$$
E_{k+1}(x)=\left\{\xi \in \mathbb{S}^{n-1}: \xi^{\prime} Q(x) \xi=\lambda_{k+1}(x), \xi \cdot \mathbf{v}_{j}(x)=0,1 \leq j \leq k\right\} .
$$

The measurability of $\lambda_{k+1}$ and $\mathbf{v}_{k+1}$ follows as above. Note also that $\lambda_{k} \in L_{l o c}^{1}(\Omega)$.

Then for any $\mathbf{f} \in \mathcal{L}^{2}(\Omega, \mathcal{Q})$ (which we now view as a representative of an equivalence class) we let $f_{j}(x)$ be the components of $\mathbf{f}(x)$ with respect to the basis of eigenvectors $\left\{\mathbf{v}_{j}(x)\right\}_{j=1}^{n}$, i.e. $\mathbf{f}(x)=\sum_{j=1}^{n} f_{j}(x) \mathbf{v}_{j}(x), x \in \Omega$. The key property for us is

$\mathcal{Q}(x, \mathbf{f}(x))=\mathbf{f}(x)^{\prime} Q(x) \mathbf{f}(x)=\left(\sum_{j=1}^{n} f_{j} \mathbf{v}_{j}\right) \cdot\left(\sum_{j=1}^{n} f_{j} Q \mathbf{v}_{j}\right)=\sum_{j=1}^{n}\left|f_{j}(x)\right|^{2} \lambda_{j}(x)$,

for $x \in \Omega$. Let $\left\|f_{j}\right\|_{L^{2}\left(\lambda_{j}\right)}=\left\{\int_{\Omega}\left|f_{j}(x)\right|^{2} \lambda_{j}(x) d x\right\}^{\frac{1}{2}}$ denote the norm of $f_{j}$ in the $L^{2}$-space on $\Omega$ with weight $\lambda_{j}$ so that (9) yields

$$
\|\mathbf{f}\|_{\mathcal{L}^{2}(\Omega, \mathcal{Q})}^{2}=\sum_{j=1}^{n}\left\|f_{j}\right\|_{L^{2}\left(\lambda_{j}\right)}^{2} .
$$

From (10) we have the following characterization of null elements in $\mathcal{L}^{2}(\Omega, \mathcal{Q})$.

Remark 6. Two functions $\mathbf{f}=\sum_{j=1}^{n} f_{j} \mathbf{v}_{j}$ and $\mathbf{g}=\sum_{j=1}^{n} g_{j} \mathbf{v}_{j}$ are in the same equivalence class in $\mathcal{L}^{2}(\Omega, \mathcal{Q})$ if and only if $f_{j}(x)=g_{j}(x)$ for $\lambda_{j}$-a.e. $x$ in $\Omega$, $1 \leq j \leq n$.

We can now mimic the standard proof of completeness of scalar-valued $L^{2}$.

Proof of Theorem 4. First we show completeness of $\mathcal{L}^{2}(\Omega, \mathcal{Q})$. Let $\left\{\mathbf{f}^{k}\right\}_{k=1}^{\infty}$ be a Cauchy sequence in $\mathcal{L}^{2}(\Omega, \mathcal{Q})$, i.e. $\left\|\mathbf{f}^{k}-\mathbf{f}^{\ell}\right\|_{\mathcal{L}^{2}(\Omega, \mathcal{Q})} \rightarrow 0$ as $k, \ell \rightarrow \infty$. Choose a rapidly converging subsequence, which we continue to label $\left\{\mathbf{f}^{k}\right\}_{k=1}^{\infty}$; i.e. $\sum_{k=1}^{\infty}\left\|\mathbf{f}^{k+1}-\mathbf{f}^{k}\right\|_{\mathcal{L}^{2}(\Omega, \mathcal{Q})}<\infty$. Let $\mathbf{f}^{k}=\sum_{j=1}^{n} f_{j}^{k} \mathbf{v}_{j}$ for $k \geq 1$. Fix $j$ for the moment and define

$$
g_{j}(x)=\left|f_{j}^{1}(x)\right|+\sum_{k=1}^{\infty}\left|f_{j}^{k+1}(x)-f_{j}^{k}(x)\right|, \quad x \in \Omega,
$$

a Lebesgue measurable function from $\Omega$ to $[0, \infty]$. From the monotone convergence theorem and Minkowski's inequality we get

$$
\begin{aligned}
\left\|g_{j}\right\|_{L^{2}\left(\lambda_{j}\right)} & =\lim _{\ell \rightarrow \infty}\left\|\left|f_{j}^{1}\right|+\sum_{k=1}^{\ell}\left|f_{j}^{k+1}-f_{j}^{k}\right|\right\|_{L^{2}\left(\lambda_{j}\right)} \\
& \leq \liminf _{\ell \rightarrow \infty}\left\{\left\|f_{j}^{1}\right\|_{L^{2}\left(\lambda_{j}\right)}+\sum_{k=1}^{\ell}\left\|f_{j}^{k+1}-f_{j}^{k}\right\|_{L^{2}\left(\lambda_{j}\right)}\right\},
\end{aligned}
$$

which equals

$$
\left\|f_{j}^{1}\right\|_{L^{2}\left(\lambda_{j}\right)}+\sum_{k=1}^{\infty}\left\|f_{j}^{k+1}-f_{j}^{k}\right\|_{L^{2}\left(\lambda_{j}\right)} \leq\left\|\mathbf{f}^{1}\right\|_{\mathcal{L}^{2}(\Omega, \mathcal{Q})}+\sum_{k=1}^{\infty}\left\|\mathbf{f}^{k+1}-\mathbf{f}^{k}\right\|_{\mathcal{L}^{2}(\Omega, \mathcal{Q})}<\infty
$$


where the final inequality follows from (10). This shows by Chebyshev's inequality that $g_{j}(x)$ is finite for $\lambda_{j}$-almost every $x \in \Omega$.

Thus the series

$$
f_{j}^{1}(x)+\sum_{k=1}^{\infty}\left(f_{j}^{k+1}(x)-f_{j}^{k}(x)\right)
$$

is absolutely convergent for $\lambda_{j}$-almost every $x \in \Omega$. Now use (11) to define

$$
f_{j}(x)= \begin{cases}f_{j}^{1}(x)+\sum_{k=1}^{\infty}\left(f_{j}^{k+1}(x)-f_{j}^{k}(x)\right) & \text { if (11) converges } \\ 0 & \text { otherwise. }\end{cases}
$$

Then $f_{j}$ is finite and Lebesgue measurable in $\Omega$, and the set where the series for $f_{j}$ diverges has $\lambda_{j}$-measure zero. Thus we have from (10),

$$
\begin{aligned}
\left\|f_{j}-f_{j}^{\ell}\right\|_{L^{2}\left(\lambda_{j}\right)} & =\left\|\sum_{k=\ell}^{\infty}\left(f_{j}^{k+1}-f_{j}^{k}\right)\right\|_{L^{2}\left(\lambda_{j}\right)} \\
& \leq \sum_{k=\ell}^{\infty}\left\|f_{j}^{k+1}-f_{j}^{k}\right\|_{L^{2}\left(\lambda_{j}\right)} \\
& \leq \sum_{k=\ell}^{\infty}\left\|\mathbf{f}^{k+1}-\mathbf{f}^{k}\right\|_{\mathcal{L}^{2}(\Omega, \mathcal{Q})} \rightarrow 0 \quad \text { as } \ell \rightarrow \infty,
\end{aligned}
$$

and so $f_{j}=f_{j}^{\ell}+\left(f_{j}-f_{j}^{\ell}\right) \in L^{2}\left(\lambda_{j}\right)$ and $f_{j}^{\ell} \rightarrow f_{j}$ in $L^{2}\left(\lambda_{j}\right)$.

Now define $\mathbf{f}(x)=\sum_{j=1}^{n} f_{j}(x) \mathbf{v}_{j}(x)$ for $x \in \Omega$. We obtain from (10) and (12) that

$$
\begin{aligned}
\left\|\mathbf{f}-\mathbf{f}^{\ell}\right\|_{\mathcal{L}^{2}(\Omega, \mathcal{Q})}^{2} & =\left\|\sum_{j=1}^{n}\left(f_{j}-f_{j}^{\ell}\right) \mathbf{v}_{j}\right\|_{\mathcal{L}^{2}(\Omega, \mathcal{Q})}^{2} \\
& =\sum_{j=1}^{n}\left\|f_{j}-f_{j}^{\ell}\right\|_{L^{2}\left(\lambda_{j}\right)}^{2} \rightarrow 0 \quad \text { as } \ell \rightarrow \infty .
\end{aligned}
$$

This shows both that $\mathbf{f}=\mathbf{f}^{\ell}+\left(\mathbf{f}-\mathbf{f}^{\ell}\right) \in \mathcal{L}^{2}(\Omega, \mathcal{Q})$ and $\mathbf{f}^{\ell} \rightarrow \mathbf{f}$ in $\mathcal{L}^{2}(\Omega, \mathcal{Q})$.

Standard arguments show that (6) defines an inner product on $\mathcal{L}^{2}(\Omega, \mathcal{Q})$ satisfying $\|\mathbf{f}\|_{\mathcal{L}^{2}(\Omega, \mathcal{Q})}=\sqrt{\langle\mathbf{f}, \mathbf{f}\rangle_{\mathcal{L}^{2}(\Omega, \mathcal{Q})}}$, and thus $\mathcal{L}^{2}(\Omega, \mathcal{Q})$ is a Hilbert space, which by (10) is isomorphic to $\bigoplus_{j=1}^{n} L^{2}\left(\lambda_{j}\right)$ under the map $\mathbf{f} \rightarrow\left(f_{1}, \ldots, f_{n}\right)$. Note that each equivalence class in $L^{2}\left(\lambda_{j}\right)$ contains a representative which is Lebesgue measurable.

3.1. The strong degenerate Sobolev space $W_{\mathcal{Q}}^{1,2}(\Omega)$. Let $\mathcal{Q}$ be a locally integrable quadratic form on $\Omega$. Define a nonnegative functional $\|w\|_{\mathcal{Q}}$ (possibly infinite) on the linear space $\operatorname{Lip}(\Omega)$ by

$$
\|w\|_{\mathcal{Q}}=\left\{\|w\|_{L^{2}(\Omega)}^{2}+\|\nabla w\|_{\mathcal{L}^{2}(\Omega, \mathcal{Q})}^{2}\right\}^{\frac{1}{2}}, \quad w \in \operatorname{Lip}(\Omega) .
$$

We then define the degenerate Sobolev space $W_{\mathcal{Q}}^{1,2}(\Omega)$ as the completion of the linear space

$$
\operatorname{Lip}_{\mathcal{Q}}(\Omega)=\left\{w \in \operatorname{Lip}(\Omega):\|w\|_{\mathcal{Q}}<\infty\right\}
$$

in the metric $d(w, v)=\|w-v\|_{\mathcal{Q}}$. 
Remark 7. In the case that $\mathcal{Q}$ and $\Omega$ are bounded, we can equivalently define $W_{\mathcal{Q}}^{1,2}(\Omega)$ as the completion of $C^{1}(\Omega)$ in the metric $d(w, v)=\|w-v\|_{\mathcal{Q}}$. Indeed, this follows immediately from the fact that $C^{1}(\Omega)$ is dense in the classical Sobolev space $H^{1,2}(\Omega)$, so that given $w \in \operatorname{Lip}(\Omega) \subset H^{1,2}(\Omega)$ and $\varepsilon>0$, we can find $v \in C^{1}(\Omega)$ with

$$
\|v-w\|_{W_{\mathcal{Q}}^{1,2}(\Omega)} \leq C\|v-w\|_{H^{1,2}(\Omega)}<\varepsilon .
$$

By construction, $W_{\mathcal{Q}}^{1,2}(\Omega)$ is a Banach space of equivalence classes of Cauchy sequences in $\operatorname{Lip}_{\mathcal{Q}}(\Omega)$. If $\mathrm{W}=\left\{w_{k}\right\}_{k=1}^{\infty}$ is a Cauchy sequence of $\operatorname{Lip}_{\mathcal{Q}}(\Omega)$ functions, i.e. $w_{k} \in \operatorname{Lip}_{\mathcal{Q}}(\Omega)$ and

$$
\left\|w_{k}-w_{\ell}\right\|_{W_{\mathcal{Q}}^{1,2}(\Omega)} \rightarrow 0 \quad \text { as } k, \ell \rightarrow \infty
$$

then there are elements (depending only on the equivalence class in $\left.W_{\mathcal{Q}}^{1,2}(\Omega)\right) w \in$ $L^{2}(\Omega)$ and $\mathbf{v} \in \mathcal{L}^{2}(\Omega, \mathcal{Q})$ such that $w_{k} \rightarrow w$ in $L^{2}(\Omega)$ and $\nabla w_{k} \rightarrow \mathbf{v}$ in $\mathcal{L}^{2}(\Omega, \mathcal{Q})$. The pair $(w, \mathbf{v}) \in L^{2}(\Omega) \times \mathcal{L}^{2}(\Omega, \mathcal{Q})$ represents the equivalence class containing the Cauchy sequence $\mathrm{W}$ in the space $W_{\mathcal{Q}}^{1,2}(\Omega)$ and provides a Hilbert space isomorphism from $W_{\mathcal{Q}}^{1,2}(\Omega)$ to a closed subspace $\mathcal{W}_{\mathcal{Q}}^{1,2}(\Omega)$ of $L^{2}(\Omega) \times \mathcal{L}^{2}(\Omega, \mathcal{Q})$ by sending the equivalence class of $\mathrm{w}$ to $(w, \mathbf{v})$. It is this realization $\mathcal{W}_{\mathcal{Q}}^{1,2}(\Omega)$ of the degenerate Sobolev space $W_{\mathcal{Q}}^{1,2}(\Omega)$ that we will use most often in the general setting.

However, the vector-valued function $\mathbf{v} \in \mathcal{L}^{2}(\Omega, \mathcal{Q})$ is not in general uniquely determined by $w \in L^{2}(\Omega)$ if $(w, \mathbf{v}) \in \mathcal{W}_{\mathcal{Q}}^{1,2}(\Omega)$. In other words, if $P$ is the Hilbert space projection of $L^{2}(\Omega) \times \mathcal{L}^{2}(\Omega, \mathcal{Q})$ onto $L^{2}(\Omega)$, then the restriction of $P$ to $\mathcal{W}_{\mathcal{Q}}^{1,2}(\Omega)$ is not in general one-to-one. Indeed, as observed in [10, an example in [2] exhibits a quadratic form $\mathcal{Q}(x, \xi)=q(x) \xi^{2}$, where $0<q(x) \leq 1, x \in \Omega=(0,1)$, $\xi \in \mathbb{R}$, together with a sequence $\left\{w_{k}\right\}_{k=1}^{\infty}$ of Lipschitz functions on $(0,1)$ such that $w_{k} \rightarrow 0$ in $L^{2}(0,1)$ (in fact $w_{k} \rightarrow 0$ uniformly on $(0,1)$ ) and $w_{k}^{\prime} \rightarrow 1$ in $\mathcal{L}^{2}((0,1), \mathcal{Q})=L^{2}((0,1), q)$. In fact, uniqueness fails for this example in the most spectacular way possible. If $\varphi \in \operatorname{Lip}_{\mathcal{Q}}(0,1)$, one sees from the calculus for $\mathcal{W}_{\mathcal{Q}}^{1,2}(\Omega)$ in Lemma 21 below that $\varphi w_{k} \rightarrow 0$ in $L^{2}(0,1)$ and $\left(\varphi w_{k}\right)^{\prime}=\varphi^{\prime} w_{k}+\varphi w_{k}^{\prime} \rightarrow \varphi$ in $\mathcal{L}^{2}((0,1), \mathcal{Q})$. Thus $(0, \varphi) \in \mathcal{W}_{\mathcal{Q}}^{1,2}(0,1)$ for all $\varphi \in \operatorname{Lip}_{\mathcal{Q}}(0,1)$, and since $\mathcal{W}_{\mathcal{Q}}^{1,2}(0,1)$ is closed, $(0, v) \in \mathcal{W}_{\mathcal{Q}}^{1,2}(0,1)$ for all $v \in \mathcal{L}^{2}((0,1), \mathcal{Q})$. Thus for each element $(u, v) \in \mathcal{W}_{\mathcal{Q}}^{1,2}(0,1)$, the fibre above $u$ consists of all of $\mathcal{L}^{2}((0,1), \mathcal{Q})$.

Note that this defect is not shared by the degenerate Sobolev space $H_{\mathcal{X}}^{1,2}(\Omega)$ when $\mathcal{X}=\left\{X_{j}\right\}_{j=1}^{m}$ is a collection of $H_{\text {div }}^{1,2}(\Omega)$ vector fields on $\Omega \subset \mathbb{R}^{n}$, since the weak derivatives defined in (3) above are unique if they exist.

We will use the notation $\mathcal{W}_{\mathcal{X}}^{1,2}(\Omega)$ and $\mathcal{L}^{2}(\Omega, \mathcal{X})$ to denote the spaces $\mathcal{W}_{\mathcal{Q}}^{1,2}(\Omega)$ and $\mathcal{L}^{2}(\Omega, \mathcal{Q})$, respectively, in case the quadratic form $\mathcal{Q}$ arises from a collection $\mathcal{X}$ of vector fields, i.e., in case $\mathcal{Q}(x, \xi)=\mathcal{X}(x, \xi)=\sum_{j=1}^{m}\left(\mathbf{v}_{j}(x) \cdot \xi\right)^{2}$ where $\mathcal{X}=$ $\left\{X_{j}\right\}_{j=1}^{m}$ and $X_{j}=\mathbf{v}_{j}(x) \cdot \nabla$.

Lemma 8. If $\mathcal{X}$ is a collection of $H_{\mathrm{div}}^{1,2}(\Omega)$ vector fields on $\Omega \subset \mathbb{R}^{n}$, then the Hilbert space projection

$$
P: L^{2}(\Omega) \times \mathcal{L}^{2}(\Omega, \mathcal{X}) \rightarrow L^{2}(\Omega)
$$

is one-to-one on $\mathcal{W}_{\mathcal{X}}^{1,2}(\Omega)$. 
Proof. If $\left\{w_{k}\right\}_{k=1}^{\infty} \subset \operatorname{Lip}_{\mathcal{X}}(\Omega)$ converges to $(0, \mathbf{u}) \in \mathcal{W}_{\mathcal{X}}^{1,2}(\Omega)$, then with $\mathcal{X}(x, \xi)=$ $\sum_{j=1}^{m}\left(\mathbf{v}_{j}(x) \cdot \xi\right)^{2}$ where $X_{j}=\mathbf{v}_{j}(x) \cdot \nabla$, we have

$$
\begin{aligned}
0 & =\lim _{k \rightarrow \infty} \int_{\Omega} \mathcal{X}\left(x, \mathbf{u}(x)-\nabla w_{k}(x)\right) d x \\
& =\lim _{k \rightarrow \infty} \int_{\Omega}\left(\sum_{j=1}^{m}\left|\mathbf{v}_{j}(x) \cdot \mathbf{u}(x)-X_{j} w_{k}(x)\right|^{2}\right) d x
\end{aligned}
$$

Thus $X_{j} w_{k} \rightarrow \mathbf{v}_{j} \cdot \mathbf{u}$ in $L^{2}(\Omega)$ for $1 \leq j \leq m$, and since $w_{k} \rightarrow 0$ in $L^{2}(\Omega)$, it follows from Theorem 2 that $\mathbf{v}_{j} \cdot \mathbf{u}$ is the weak derivative $X_{j} 0$ of 0 . Uniqueness of weak derivatives shows that $\mathbf{v}_{j} \cdot \mathbf{u}=0$ a.e. for $1 \leq j \leq m$, and thus $\mathbf{u}=0$ in $\mathcal{L}^{2}(\Omega, \mathcal{X})$ since $\int_{\Omega} \mathcal{X}(x, \mathbf{u}(x)) d x=\int_{\Omega}\left(\sum_{j=1}^{m}\left|\mathbf{v}_{j}(x) \cdot \mathbf{u}(x)\right|^{2}\right) d x=0$.

In particular, this lemma applies to a quadratic form $\mathcal{Q}$ when the rows $\mathbf{v}_{1}(x), \ldots$, $\mathbf{v}_{n}(x)$ of the matrix $\sqrt{Q(x)}$ are in $H_{\text {div }}^{1,2}(\Omega)$, since then $\mathcal{Q}(x, \xi)=\sum_{k=1}^{m}\left|\mathbf{v}_{k}(x) \cdot \xi\right|^{2}$.

Remark 9 . For $1 \leq p<\infty$ and $\mathcal{Q}$ locally integrable, we can also define the analogous degenerate Sobolev space $W_{\mathcal{Q}}^{1, p}(\Omega)$ with strong derivatives in $L^{p}$ as the completion of $\operatorname{Lip}_{\mathcal{Q}, p}(\Omega)=\left\{w \in \operatorname{Lip}(\Omega):\|w\|_{\mathcal{Q}, p}<\infty\right\}$ where

$$
\|w\|_{\mathcal{Q}, p}=\left(\int_{\Omega}|w|^{p}+\int_{\Omega}\left[(\nabla w)^{\prime} Q(\nabla w)\right]^{\frac{p}{2}}\right)^{\frac{1}{p}} .
$$

3.2. Comparison of spaces $H_{\mathcal{X}}^{1,2}(\Omega)$ and $W_{\mathcal{Q}}^{1,2}(\Omega)$. As mentioned in the introduction, the question now arises as to the equality of the two degenerate Sobolev spaces $H_{\mathcal{X}}^{1,2}(\Omega)$ and $W_{\mathcal{Q}}^{1,2}(\Omega)$ when $\mathcal{X}=\left\{X_{j}\right\}_{j=1}^{m}, X_{j}=\mathbf{v}_{j}(x) \cdot \nabla$ is a collection of $H_{\text {div }}^{1,2}(\Omega)$ vector fields on $\Omega \subset \mathbb{R}^{n}$, and the forms $\mathcal{Q}(x, \xi)$ and $\mathcal{X}(x, \xi)=$ $\sum_{j=1}^{m}\left(\mathbf{v}_{j}(x) \cdot \xi\right)^{2}$ are comparable:

$$
\mathcal{Q}(x, \xi) \approx \mathcal{X}(x, \xi)
$$

Clearly $W_{\mathcal{Q}}^{1,2}(\Omega)=W_{\mathcal{X}}^{1,2}(\Omega)$ when (15) holds. The map $j: \operatorname{Lip} p_{\mathcal{X}}(\Omega) \rightarrow W_{\mathcal{X}}^{1,2}(\Omega)$ that sends $w$ to the constant sequence $\{w\}$ is an isometry with the norm $\|\cdot\|_{\mathcal{X}}$ on $\operatorname{Lip}_{\mathcal{X}}(\Omega)$. The inverse map $i$ takes $j\left(\operatorname{Lip}_{\mathcal{X}}(\Omega)\right)$ isometrically onto $\operatorname{Lip}_{\mathcal{X}}(\Omega)$ in the Hilbert space $H_{\mathcal{X}}^{1,2}(\Omega)$. Since $j(\operatorname{Lip} \mathcal{X}(\Omega))$ is dense in $W_{\mathcal{X}}^{1,2}(\Omega)$, and $H_{\mathcal{X}}^{1,2}(\Omega)$ is complete, the map $i$ has a unique continuous extension

$$
i: W_{\mathcal{X}}^{1,2}(\Omega) \rightarrow H_{\mathcal{X}}^{1,2}(\Omega)
$$

which is an isometry that we loosely refer to as an inclusion $W_{\mathcal{X}}^{1,2}(\Omega) \subset H_{\mathcal{X}}^{1,2}(\Omega)$.

As we observed above, gradients of elements in $W_{\mathcal{X}}^{1,2}(\Omega)$ are uniquely determined in this case; i.e., the projection $P$ above is one-to-one when restricted to $\mathcal{W}_{\mathcal{X}}^{1,2}(\Omega)$. By contrast, recall for a moment the counterexample from [2] that we described in the previous subsection. The Lipschitz function 0 is represented by the pair $(0,0)$ in $\mathcal{W}_{\mathcal{Q}}^{1,2}(\Omega)$, while the Cauchy sequence $\left\{w_{k}\right\}_{k=1}^{\infty}$ from the counterexample is represented by the pair $(0,1)$. Of course the function $q(x)$ in the quadratic form $\mathcal{Q}$ in the counterexample fails to have a square integrable derivative, which allows for the failure of injectivity of $P$ when restricted to $\mathcal{W}_{\mathcal{Q}}^{1,2}(\Omega)$. 
In the case when $\mathcal{X}$ is a collection of $H_{\text {div }}^{1,2}(\Omega)$ vector fields, we showed above that a pair $(w, \mathbf{v}) \in \mathcal{W}_{\mathcal{X}}^{1,2}(\Omega)$ is uniquely determined by its $L^{2}$ component $w$. Thus we may here also realize $W_{\mathcal{X}}^{1,2}(\Omega)$ as a (not necessarily closed) subspace $\mathrm{W}_{\mathcal{X}}^{1,2}(\Omega)$ of $L^{2}(\Omega)$, and we may unambiguously write $\nabla w$ for the element $\mathbf{v} \in \mathcal{L}^{2}(\Omega, \mathcal{X})$. As a result, we typically write $(w, \nabla w)$ for the element $(w, \mathbf{v})$ of $\mathcal{W}_{\mathcal{X}}^{1,2}(\Omega)$, and in this way we see that the gradients $\nabla w$ of $L^{2}$ functions $w$ in $W_{\mathcal{X}}^{1,2}(\Omega)$ live in the form-weighted space $\mathcal{L}^{2}(\Omega, \mathcal{X})$. Note that this form-weighted space, and so also the meaning of $\nabla w$, depends on $\mathcal{X}$.

Remark 10. We have introduced separate notation for the three different realizations of the degenerate space $W_{\mathcal{Q}}^{1,2}(\Omega)$. We write $\mathrm{w} \in W_{\mathcal{Q}}^{1,2}(\Omega)$ to denote an equivalence class of Cauchy sequences in the definition by completion, $(w, \mathbf{v}) \in \mathcal{W}_{\mathcal{Q}}^{1,2}(\Omega)$ to denote the $L^{2}(\Omega)$ component $w$ and the $\mathcal{L}^{2}(\Omega, \mathcal{Q})$ component $\mathbf{v}$ associated with $\mathrm{W}$, and finally in the case $\mathcal{Q}=\mathcal{X}$, we write $w \in \mathrm{W}_{\mathcal{X}}^{1,2}(\Omega)$ to denote the $L^{2}(\Omega)$ component which now uniquely determines the gradient component. We caution the reader that for an element $\mathrm{W}$ in $W_{\mathcal{Q}}^{1,2}(\Omega)$ represented by $(w, \mathbf{v}) \in \mathcal{W}_{\mathcal{Q}}^{1,2}(\Omega)$, we often abuse notation by writing $w$ in place of $\mathrm{w}$, and $\nabla w$ in place of $\mathbf{v}$, with the understanding that $\nabla w$ is not in general uniquely determined by $w$. In particular, we write $w=\lim _{k \rightarrow \infty} w_{k}$ in $W_{\mathcal{Q}}^{1,2}(\Omega)$ if $\mathrm{w}=\left\{w_{k}\right\}_{k=1}^{\infty} \in W_{\mathcal{Q}}^{1,2}(\Omega)$.

It is a result of Franchi, Serapioni and Serra Cassano 3, and independently Garofalo and Nhieu [4, that the degenerate Sobolev spaces $W_{\mathcal{X}}^{1,2}(\Omega)$ and $H_{\mathcal{X}}^{1,2}(\Omega)$ coincide when the vector fields $\mathcal{X}=\left\{X_{j}\right\}_{j=1}^{m}$ are Lipschitz. We show later that $H_{\mathcal{X}}^{1,2}(\Omega)=W_{\mathcal{X}}^{1,2}(\Omega)$ if the vector fields $\mathcal{X}=\left\{X_{j}\right\}_{j=1}^{m}$ are in

$$
H^{1,2 \sigma^{\prime}}(\Omega)=\left\{v \in L^{2 \sigma^{\prime}}(\Omega): \nabla v \in L^{2 \sigma^{\prime}}(\Omega)\right\},
$$

where $\frac{1}{\sigma}+\frac{1}{\sigma^{\prime}}=1$, and $\sigma \geq 1$ is such that the following Sobolev inequality holds:

$$
\left\{\int_{\omega}|w|^{2 \sigma}\right\}^{\frac{1}{2 \sigma}} \leq C_{\omega}\left\{\int_{\omega}|w|^{2}+\int_{\omega}|\mathcal{X} w|^{2}\right\}^{\frac{1}{2}}
$$

for all open sets $\omega \Subset \Omega$ and for all $w \in \operatorname{Lip}_{c}(\omega)$. Alternatively, we can relax the regularity assumption on $\mathcal{X}$ to $X_{j} \in H_{\text {div }}^{1,2}(\Omega)$ provided $\mathcal{X}$ is comparably Lipschitz off its common zero set $Z$, and Lipschitz at $Z$. Moreover, $H_{\mathcal{X}}^{1,2}(\Omega)=W_{\mathcal{X}}^{1,2}(\Omega)$ in dimension $n=1$ without any restriction on the vector fields other than the assumption $\mathcal{X} \in H_{\text {div }}^{1,2}(\Omega)$, which is necessary for the definition of $H_{\mathcal{X}}^{1,2}(\Omega)$.

Finally, we turn our attention briefly to the case $1<p<\infty$. If $\mathcal{X}=\left\{X_{j}\right\}_{j=1}^{m} \in$ $H_{\text {div }}^{1, p^{\prime}}(\Omega)$ satisfies $\mathcal{X}(x, \xi) \approx \mathcal{Q}(x, \xi)$, then

$$
\sum_{j=1}^{m}\left|X_{j} w(x)\right|^{p} \approx\left(\sum_{j=1}^{m}\left|X_{j} w(x)\right|^{2}\right)^{\frac{p}{2}}=\mathcal{X}(x, \nabla w(x))^{\frac{p}{2}} \approx \mathcal{Q}(x, \nabla w(x))^{\frac{p}{2}}
$$

for $w \in \operatorname{Lip}_{\mathcal{Q}, p}(\Omega)$. Thus $\|w\|_{\mathcal{Q}, p} \approx\|w\|_{H_{\mathcal{X}}^{1, p}(\Omega)}$ for $w \in \operatorname{Lip}_{\mathcal{Q}, p}(\Omega)$ and if, as in the case $p=2$ above, we identify $\operatorname{Lip}_{\mathcal{Q}, p}(\Omega)$ with the space of corresponding constant sequences in $W_{\mathcal{Q}}^{1, p}(\Omega)$, then $W_{\mathcal{Q}}^{1, p}(\Omega)$ is isomorphic to the closure of $\operatorname{Lip}_{\mathcal{Q}, p}(\Omega)$ in $H_{\mathcal{X}}^{1, p}(\Omega)$ (see Remark 3 for the definition of $H_{\mathcal{X}}^{1, p}(\Omega)$ ). Now it is still true that 
$H_{\mathcal{X}}^{1, p}(\Omega)=W_{\mathcal{X}}^{1, p}(\Omega)$ for $\mathcal{X}$ Lipschitz $\left([3],[4)\right.$, but it may happen that $H_{\mathcal{X}}^{1, p}(\Omega) \neq$ $W_{\mathcal{X}}^{1, p}(\Omega)$ for certain $\mathcal{X} \in H_{\text {div }}^{1, p^{\prime}}(\Omega)$ when $p>2$. In fact we show that in dimension $n=1, H_{\mathcal{X}}^{1, p}(\Omega)=W_{\mathcal{X}}^{1, p}(\Omega)$ for all $\mathcal{X} \in H^{1, p^{\prime}}(\Omega)$ when $1<p \leq 2$, while for $p>2$ in all dimensions, there is for every $p^{\prime} \leq q<p$, a vector field $\mathcal{X} \in H^{1, q}(\Omega)$ such that $H_{\mathcal{X}}^{1, p}(\Omega) \neq W_{\mathcal{X}}^{1, p}(\Omega)$. See Section 5 below on equality of degenerate Sobolev spaces for all of the results described here.

3.3. $W_{\mathcal{Q}}$-weak solutions of degenerate elliptic equations. Let $\Omega$ be a bounded open subset of $\mathbb{R}^{n}$. Consider the linear differential operator

$$
\mathcal{L} \equiv L+\mathbf{H R}+\mathbf{S}^{\prime} \mathbf{G}+F
$$

in $\Omega$, and the equation

$$
\mathcal{L} u=f+\mathbf{T}^{\prime} \mathbf{g},
$$

where $\mathbf{R}=\left\{R_{i}\right\}_{i=1}^{N}, \mathbf{S}=\left\{S_{i}\right\}_{i=1}^{N}$ and $\mathbf{T}$ are collections of vector fields subunit with respect to $Q(x)\left(S=\mathbf{v} \cdot \nabla\right.$ is subunit if $\left.(\mathbf{v} \cdot \xi)^{2} \leq \xi^{\prime} Q \xi\right)$, and $F, \mathbf{G}=\left\{G_{i}\right\}_{i=1}^{N}$ and $\mathbf{H}=\left\{H_{i}\right\}_{i=1}^{N}$ are bounded measurable functions, and the inhomogeneous data $f$ and $\mathbf{g}$ are in $L^{2}(\Omega)$. Here the juxtaposition of vectors in HR, GS and gT means $\sum_{i=1}^{N} H_{i} R_{i}, \sum_{i=1}^{N} G_{i} S_{i}$ and $\sum_{i=1}^{N} g_{i} T_{i}$, respectively, and the prime ' denotes transpose, so that e.g. $(\mathbf{G S})^{\prime}=\mathbf{S}^{\prime} \mathbf{G}$ and $(\mathbf{g T})^{\prime}=\mathbf{T}^{\prime} \mathbf{g}$.

We now give the definition of a $W_{\mathcal{Q}}$-weak solution $u$ to equation (17). First, for $u \in W_{\mathcal{Q}}^{1,2}(\Omega)$ we write $(u, \nabla u) \in \mathcal{W}_{\mathcal{Q}}^{1,2}(\Omega)$ with the understanding that $\nabla u$ is not uniquely determined by $u$, i.e. $\nabla u$ denotes one of the vector-valued functions in $\mathcal{L}^{2}(\Omega, \mathcal{Q})$ for which $(u, \nabla u) \in \mathcal{W}_{\mathcal{Q}}^{1,2}(\Omega)$. Then for $u, w \in W_{\mathcal{Q}}^{1,2}(\Omega)$ the expression $\nabla u^{\prime} Q \nabla w$ is well defined as an integrable function in $\Omega$ since

$$
\begin{aligned}
\left\|\nabla u^{\prime} Q \nabla w\right\|_{L^{1}(\Omega)} & =\int_{\Omega}\left|\nabla u^{\prime}(x) Q(x) \nabla w(x)\right| d x \\
& \leq \int_{\Omega} \sqrt{\mathcal{Q}(x, \nabla u(x))} \sqrt{\mathcal{Q}(x, \nabla w(x))} d x \\
& \leq\|\nabla u\|_{\mathcal{L}^{2}(\Omega, \mathcal{Q})}\|\nabla w\|_{\mathcal{L}^{2}(\Omega, \mathcal{Q})} .
\end{aligned}
$$

Next we note that if $T=\mathbf{a} \cdot \nabla$ is subunit with respect to $Q(x)$ and $u \in W_{\mathcal{Q}}^{1,2}(\Omega)$, then $T u=\mathbf{a} \cdot \nabla u$ is well defined as a square integrable function in $\Omega$ since

$$
\begin{aligned}
\|T u\|_{L^{2}(\Omega)}^{2} & =\int_{\Omega}|\mathbf{a} \cdot \nabla u(x)|^{2} d x \\
& \leq \int_{\Omega} \mathcal{Q}(x, \nabla u(x)) d x \\
& \leq\|\nabla u\|_{\mathcal{L}^{2}(\Omega, \mathcal{Q})} .
\end{aligned}
$$

We are now ready for the definition of $W_{\mathcal{Q}}$-weak solution. Note that by the discussion above, all of the integrals appearing below are absolutely convergent for $u, w \in W_{\mathcal{Q}}^{1,2}(\Omega)$, vector fields $\mathbf{R}, \mathbf{S}, \mathbf{T}$ subunit with respect to $Q$, vector functions $\mathbf{G}, \mathbf{H}$ bounded and $f, \mathbf{g} \in L^{2}(\Omega)$. We define $\operatorname{Lip}_{c}(\Omega)$ to consist of the Lipschitz functions with compact support in $\Omega$. 
Definition 11. An element $(u, \nabla u) \in \mathcal{W}_{\mathcal{Q}}^{1,2}(\Omega)$ is a $W_{\mathcal{Q}}$-weak $\left(\begin{array}{c}\text { solution } \\ \text { subsolution } \\ \text { supersolution }\end{array}\right)$ of (17) in $\Omega$ if

$$
-\int(\nabla u)^{\prime} Q \nabla w+\int(\mathbf{H R} u) w+\int u \mathbf{G S} w+\int F u w\left(\begin{array}{c}
= \\
\geq \\
\leq
\end{array}\right) \int f w+\int \mathbf{g} \mathbf{T} w,
$$

for all nonnegative $w \in \operatorname{Lip}_{c}(\Omega)$.

Equivalently, we could test over all nonnegative $w \in\left(W_{\mathcal{Q}}^{1,2}\right)_{0}(\Omega)$, the closure of $\operatorname{Lip}_{c}(\Omega)$ in $W_{\mathcal{Q}}^{1,2}(\Omega)$; we say that an element $(u, \nabla u) \in \mathcal{W}_{\mathcal{Q}}^{1,2}(\Omega)$ is nonnegative if $u \geq 0$.

Recall the element $(0,1)$ in $\mathcal{W}_{\mathcal{Q}}^{1,2}(0,1)$ discussed earlier that arose from the example in [2]. This element is a $W_{\mathcal{Q}}$-weak solution of the equation $\frac{d}{d x} q(x) \frac{d}{d x} u=T^{\prime} 1$ where $T=-q(x) \frac{d}{d x}$ is a bounded subunit vector field. Indeed, the corresponding integral equality is

$$
-\int\left(\frac{d}{d x} u\right)(x) q(x)\left(\frac{d}{d x} w\right)(x) d x=-\int 1 \cdot q(x)\left(\frac{d}{d x} w\right)(x) d x,
$$

which holds since $\frac{d}{d x} u=1$ in $\mathcal{L}^{2}((0,1), \mathcal{Q})$ for the element $u=(0,1)$. On the other hand, the zero element $(0,0) \in \mathcal{W}_{\mathcal{Q}}^{1,2}(0,1)$ is not a weak solution of the equation $\frac{d}{d x} q(x) \frac{d}{d x} u=T^{\prime} 1$.

Remark 12. Alternatively, as is done in [10] for $u, w \in W_{\mathcal{Q}}^{1,2}(\Omega)$ and $T$ subunit, we can define

$$
\nabla u^{\prime} Q \nabla w \in L^{1}(\Omega) \text { and } T u \in L^{2}(\Omega)
$$

solely by reference to Cauchy sequences of elements in $\operatorname{Lip}_{\mathcal{Q}}(\Omega)$, without using $\mathcal{L}^{2}(\Omega, \mathcal{Q})$. Let $u=\lim _{k \rightarrow \infty} u_{k}$ and $w=\lim _{k \rightarrow \infty} w_{k}$ in $W_{\mathcal{Q}}^{1,2}(\Omega)$ where $u_{k}, w_{k} \in$ $\operatorname{Lip}_{\mathcal{Q}}(\Omega)$. Then $\left\{\nabla u_{k}^{\prime} Q \nabla w_{k}\right\}_{k=1}^{\infty}$ is Cauchy in $L^{1}(\Omega)$ using

$$
\begin{aligned}
& \int_{\Omega}\left|\nabla u_{k}^{\prime} Q \nabla w_{k}-\nabla u_{\ell}^{\prime} Q \nabla w_{\ell}\right| \\
& \leq \int_{\Omega}\left|\left(\nabla u_{k}-\nabla u_{\ell}\right)^{\prime} Q \nabla w_{k}\right|+\int_{\Omega}\left|\nabla u_{\ell}^{\prime} Q\left(\nabla w_{k}-\nabla w_{\ell}\right)\right| \\
& \leq\left\{\int_{\Omega} \mathcal{Q}\left(x, \nabla u_{k}(x)-\nabla u_{\ell}(x)\right)\right\}^{\frac{1}{2}}\left\{\int_{\Omega} \mathcal{Q}\left(x, \nabla w_{k}(x)\right)\right\}^{\frac{1}{2}} \\
& +\left\{\int_{\Omega} \mathcal{Q}\left(x, \nabla u_{\ell}(x)\right)\right\}^{\frac{1}{2}}\left\{\int_{\Omega} \mathcal{Q}\left(x, \nabla w_{k}(x)-\nabla w_{\ell}(x)\right)\right\}^{\frac{1}{2}} .
\end{aligned}
$$

It follows that there is a unique element $\nabla u^{\prime} Q \nabla w \in L^{1}(\Omega)$ that satisfies

$$
\begin{aligned}
\nabla u^{\prime} Q \nabla w & =\lim _{k \rightarrow \infty} \nabla u_{k}^{\prime} Q \nabla w_{k} \text { in } L^{1}(\Omega) \text { whenever } \\
u & =\lim _{k \rightarrow \infty} u_{k} \text { and } w=\lim _{k \rightarrow \infty} w_{k} \text { in } W_{\mathcal{Q}}^{1,2}(\Omega) .
\end{aligned}
$$


Moreover, if $T=\mathbf{a} \cdot \nabla$ is subunit, then $\left\{T u_{k}\right\}_{k=1}^{\infty}$ is Cauchy in $L^{2}(\Omega)$ using

$$
\begin{aligned}
\left\|T u_{k}-T u_{\ell}\right\|_{L^{2}(\Omega)}^{2} & =\int_{\Omega}\left|\mathbf{a} \cdot\left(\nabla u_{k}-\nabla u_{\ell}\right)(x)\right|^{2} d x \\
& \leq \int_{\Omega} \mathcal{Q}\left(x, \nabla u_{k}(x)-\nabla u_{\ell}(x)\right) d x \\
& =\left\|u_{k}-u_{\ell}\right\|_{\mathcal{L}^{2}(\Omega, \mathcal{Q})}^{2} .
\end{aligned}
$$

Thus there is a unique element $T u \in L^{2}(\Omega)$ that satisfies

$$
T u=\lim _{k \rightarrow \infty} T u_{k} \text { in } L^{2}(\Omega) \text { whenever } u=\lim _{k \rightarrow \infty} u_{k} \text { in } W_{\mathcal{Q}}^{1,2}(\Omega) .
$$

\section{The Regularity theorems}

In order to state our generalization of Theorem 8 in [11, we recall some notation; see [11] for more details. A quasimetric $d$ on an open set $\Omega \subset \mathbb{R}^{n}$ is a finite nonnegative function on $\Omega \times \Omega$ satisfying

$$
\begin{aligned}
& d(x, y)=0 \Longleftrightarrow x=y, \\
& d(x, y) \leq \kappa(d(x, z)+d(y, z))
\end{aligned}
$$

for all $x, y, z$ in $\Omega$. The quasimetric balls $B(x, r)$ are defined by

$$
B(x, r)=\{y \in \Omega: d(x, y)<r\}, \quad 0<r<\infty .
$$

Provided the quasimetric $d(x, y)$ is Lebesgue measurable in the second variable (so that the balls are measurable), the upper and lower dimensions, $Q^{*}$ and $Q_{*}$, of a quasimetric space with balls $B(x, r)$ are given by

$$
\begin{aligned}
& Q^{*}=\limsup _{r \rightarrow 0} \max _{x \in \Omega} \frac{\log |B(x, r)|}{\log r}, \\
& Q_{*}=\liminf _{r \rightarrow 0} \min _{x \in \Omega} \frac{\log |B(x, r)|}{\log r} .
\end{aligned}
$$

We will require the following containment condition relative to Euclidean balls $D(x, r)$, which is essentially necessary for the notion of subellipticity of the form $\mathcal{Q}$ that is given in Definition 15 below: there are positive constants $C, \varepsilon$ and $\delta$ such that

$$
D(x, r) \subset B\left(x, C r^{\varepsilon}\right), \quad x \in \Omega, 0<r<\delta \operatorname{dist}(x, \partial \Omega) .
$$

We will also require the doubling condition

$$
|B(x, 2 r)| \leq C|B(x, r)|, \quad x \in \Omega, 0<r<\infty,
$$

which makes $(\Omega, d,|\cdot|)$ into a homogeneous space (more precisely what is called a general homogeneous space in [11), and makes $Q^{*}$ and $Q_{*}$ finite.

Given an integrable nonnegative semidefinite quadratic form $\mathcal{Q}(x, \xi)=\xi^{\prime} Q(x) \xi$, where $Q(x)$ is a symmetric matrix for each $x \in \Omega$, and an $\mathbb{R}^{n}$-valued function $\mathbf{f}$, we define

$$
|\mathbf{f}(x)|_{\mathcal{Q}}^{2}=\mathbf{f}(x)^{\prime} Q(x) \mathbf{f}(x)
$$

and assume the following Sobolev inequality: there is $\sigma>1$ and $\delta>0$ such that for all balls $B=B(y, r)$ with $y \in \Omega, 0<r<\delta$ dist $(y, \partial \Omega)$,

$$
\left\{\frac{1}{|B|} \int_{B}|w|^{2 \sigma}\right\}^{\frac{1}{2 \sigma}} \leq C r\left\{\frac{1}{|B|} \int_{B}|\nabla w|_{\mathcal{Q}}^{2}\right\}^{\frac{1}{2}}+C\left\{\frac{1}{|B|} \int_{B}|w|^{2}\right\}^{\frac{1}{2}}
$$


for all $(w, \nabla w) \in\left(\mathcal{W}_{\mathcal{Q}}^{1,2}\right)_{0}(B)$, the closure in $\mathcal{W}_{\mathcal{Q}}^{1,2}(B)$ of $(w, \nabla w)$ where $w$ is a Lipschitz function compactly supported in $\Omega$. Note that the right-hand side of (21) is comparable to the normalized $\mathcal{Q}$-Sobolev norm

$$
\|w\|_{W_{\mathcal{Q}}^{1,2}(B)}^{*} \equiv\left\{\frac{1}{|B|} \int_{B}\left(|r \nabla w|_{\mathcal{Q}}^{2}+|w|^{2}\right)\right\}^{\frac{1}{2}} .
$$

The Poincaré inequality we need is: there is $C_{0} \geq 1$ and $\delta>0$ such that for all balls $B=B(y, r)$ and $B^{*}=B\left(y, C_{0} r\right)$ with $y \in \Omega, 0<C_{0} r<\delta \operatorname{dist}(y, \partial \Omega)$,

$$
\left\{\frac{1}{|B|} \int_{B}\left|w-\left(\frac{1}{|B|} \int_{B} w\right)\right|^{2}\right\}^{\frac{1}{2}} \leq C r\left\{\frac{1}{\left|B^{*}\right|} \int_{B^{*}}|\nabla w|_{\mathcal{Q}}^{2}\right\}^{\frac{1}{2}},
$$

for every $(w, \nabla w) \in \mathcal{W}_{\mathcal{Q}}^{1,2}(B)$. The following quantity will play the role of "dimension" in the sequel:

$$
Q=\max \left\{Q^{*}, 2 \sigma^{\prime}\right\}
$$

where $\frac{1}{\sigma}+\frac{1}{\sigma^{\prime}}=1$.

Our next hypothesis (27) is crucial for Moser iteration, and holds automatically with $p=\infty$ for the subunit balls $K(x, r)$ associated to $\mathcal{Q}$, if $\mathcal{Q}(x, \xi)$ is continuous in $x$ and (19) holds for the subunit balls, i.e.

$$
D(x, r) \subset K\left(x, C r^{\varepsilon}\right), \quad x \in \Omega, 0<r<\delta \operatorname{dist}(x, \partial \Omega) .
$$

Recall that the subunit balls

$$
K(x, r)=\{y \in \Omega: \delta(x, y)<r\}
$$

are defined using the control metric

$$
\delta(x, y)=\inf \{r>0: \gamma(0)=x, \gamma(r)=y, \gamma \text { is Lipschitz and subunit }\},
$$

where $\gamma(t), 0 \leq t \leq r$, is a subunit curve in $\Omega$ if $\left(\gamma^{\prime}(t) \cdot \xi\right)^{2} \leq \mathcal{Q}(\gamma(t), \xi)$ for $0 \leq t \leq r$.

We suppose there are positive constants $c, N$ and $\delta$ such that for each ball $B(y, r)$ with $y \in \Omega, 0<r<\delta \operatorname{dist}(y, \partial \Omega)$, there is an accumulating sequence of Lipschitz cutoff functions $\left\{\psi_{j}\right\}_{j=1}^{\infty}$ on $B(y, r)$ with the following five properties ( $E \Subset F$ means that the closure of $E$ is contained in the interior of $F$ ):

$$
\left\{\begin{array}{lll}
\text { supp } \psi_{1} & \subset B(y, r), & \\
B(y, c r) & \subset\left\{x: \psi_{j}(x)=1\right\}, & j \geq 1, \\
\text { supp } \psi_{j+1} & \Subset\left\{x: \psi_{j}(x)=1\right\}, & j \geq 1, \\
\psi_{j} & \text { is Lipschitz, } & j \geq 1, \\
\left\{\frac{1}{|B(y, r)|} \int\left|\nabla \psi_{j}\right|_{\mathcal{Q}}^{p} d x\right\}^{\frac{1}{p}} & \leq C_{p} \frac{j^{N}}{r}, & j \geq 1,
\end{array}\right.
$$

for some $p>2 \sigma^{\prime}$.

We consider the subelliptic equation (17). Since we now have the Sobolev inequality (21), we can relax the requirements on the coefficients in our degenerate equation (17). Our hypothesis on the operator coefficients $\mathbf{H}, \mathbf{G}$ and $F$ is

$$
\|F\|_{L^{\frac{q}{2}(\Omega)}}+\|\mathbf{G}\|_{L^{q}(\Omega)}+\|\mathbf{H}\|_{L^{q}(\Omega)} \equiv N_{q}<\infty,
$$

for some $q>Q$. Our hypothesis on the inhomogeneous data $f, \mathbf{g}$ is

$$
\|f\|_{L^{\frac{q}{2}(\Omega)}}+\|\mathbf{g}\|_{L^{q}(\Omega)} \equiv N_{q}^{\prime}<\infty,
$$


for the same $q>Q$ as in (28). The distinction between $N_{q}$ and $N_{q}^{\prime}$ is made here because the Hölder continuity exponent $\alpha$ of weak solutions $u$ to (17) will turn out to depend on the gaps $1-\frac{Q}{p}$ and $1-\frac{Q}{q}$, where $p$ is as in (27) and $q$ is as in (28) and (29), as well as on $C_{p}$ in (27) and $N_{q}$ in (28), but not on $u$ or $N_{q}^{\prime}$ in (29). This is of paramount importance in applications to nonlinear equations - see e.g. [7].

As in Definition [11 we say that an element $(u, \nabla u) \in \mathcal{W}_{\mathcal{Q}}^{1,2}(\Omega)$ is a $W_{\mathcal{Q}}$-weak solution of (17) in $\Omega$ if $u$ and $\nabla u$ satisfy

$$
-\int(\nabla u)^{\prime} Q \nabla w+\int(\mathbf{H R} u) w+\int u \mathbf{G S} w+\int F u w=\int f w+\int \mathbf{g} \mathbf{T} w,
$$

for all $w \in \operatorname{Lip}_{c}(\Omega)$. Note as in [11] that all of the above integrals are absolutely convergent for $u, w \in W_{\mathcal{Q}}^{1,2}(\Omega)$ if we use $q>Q \geq 2 \sigma^{\prime}$ and the fact that (21) implies $u, w \in L_{\text {loc }}^{2 \sigma}(\Omega)$. As a consequence we may test the integral inequality above over all $w \in\left(W_{\mathcal{Q}}^{1,2}\right)_{0}(\Omega)$, the closure of $\operatorname{Lip}_{c}(\Omega)$ in $W_{\mathcal{Q}}^{1,2}(\Omega)$. This is of course needed to implement Moser iteration.

The next definition incorporates the new generality of our regularity theorems by requiring Hölder continuity for each $W_{\mathcal{Q}}$-weak solution of (17), rather than merely for each classical weak solution, as was assumed in [11.

Definition 13. Let $q \in[2, \infty]$. We say that an operator $L=\nabla^{\prime} Q(x) \nabla$ with locally integrable matrix $Q(x)$ is $L^{q}$-subelliptic in $\Omega$ if there are positive functions $\alpha=\alpha\left(E, z_{1}\right)$ and $\mathcal{C}=\mathcal{C}\left(E, z_{1}, z_{2}, z_{3}\right)$ defined on $\mathcal{P}(\Omega) \times[0, \infty)$ and $\mathcal{P}(\Omega) \times[0, \infty)^{3}$ respectively (where $\mathcal{P}(\Omega)$ is the lattice of compact subsets of $\Omega$ ), increasing in each variable separately, such that every $W_{\mathcal{Q}}$-weak solution $(u, \nabla u)$ of (17) in $\Omega$ satisfies, possibly after redefining $u$ on a set of measure zero,

$$
\|u\|_{C^{\alpha}(K)} \leq \mathcal{C}
$$

for

$$
\begin{aligned}
& \alpha=\alpha\left(K, N_{q}\right), \\
& \mathcal{C}=\mathcal{C}\left(K, N_{q}, N_{q}^{\prime},\|u\|_{2}\right),
\end{aligned}
$$

whenever $K$ is a compact subset of $\Omega$, (29) and (28) hold, and $\mathbf{R}=\left\{R_{i}\right\}_{i=1}^{N}$, $\mathbf{S}=\left\{S_{i}\right\}_{i=1}^{N}$ and $\mathbf{T}=\left\{T_{i}\right\}_{i=1}^{N}$ are collections of vector fields subunit with respect to $Q(x)$.

Remark 14. The conclusion (30) applies only to the $L^{2}$ component of $(u, \nabla u) \in$ $\mathcal{W}_{\mathcal{Q}}(\Omega)$ and says nothing about the associated (nonunique) gradient $\nabla u$.

With the above new definition of an $L^{q}$-subelliptic operator in terms of $W_{\mathcal{Q}}$-weak solutions, the next definition and theorems can be stated exactly as in 11.

Definition 15. Let $q \in[2, \infty]$. We say that a locally integrable nonnegative semidefinite quadratic form $\mathcal{Q}(x, \xi)$ is $L^{q}$-subelliptic in $\Omega$ if every operator $L=$ $\nabla^{\prime} B(x) \nabla$ whose matrix $B(x)$ satisfies

$$
c_{\text {sym }} \mathcal{Q}(x, \xi) \leq \xi^{\prime} B(x) \xi \leq C_{\text {sym }} \mathcal{Q}(x, \xi), \quad \text { a.e. } x \in \Omega, \xi \in \mathbb{R}^{n},
$$

for positive constants $c_{\text {sym }}$ and $C_{\text {sym }}$, is $L^{q}$-subelliptic in $\Omega$, and provided the positive functions $\alpha$ and $\mathcal{C}$ in (31) can be chosen to depend only on the constants 
$c_{\text {sym }}$ and $C_{\text {sym }}$ in (32) and not on $L$ itself, i.e.

$$
\begin{aligned}
& \alpha=\alpha_{c_{s y m}, C_{s y m}}\left(K, N_{q}\right), \\
& \mathcal{C}=\mathcal{C}_{c_{\text {sym }}, C_{s y m}}\left(K, N_{q}, N_{q}^{\prime},\|u\|_{2}\right) .
\end{aligned}
$$

Theorem 16. Suppose that $\mathcal{Q}(x, \xi)$ is a locally integrable nonnegative semidefinite quadratic form in a bounded open set $\Omega$. Let $d(x, y)$ be a symmetric quasimetric in $\Omega$ with $d(x, y) \geq c|x-y|$ for some $c>0$ that is Lebesgue measurable in each variable separately, with upper dimension $Q^{*}$, and suppose $\sigma>1$. Then $\mathcal{Q}(x, \xi)$ is $L^{q}$-subelliptic in $\Omega$ for

$$
q>Q \equiv \max \left\{Q^{*}, 2 \sigma^{\prime}\right\}, \quad \frac{1}{\sigma}+\frac{1}{\sigma^{\prime}}=1,
$$

provided that the following hold for the d-balls $B(x, r)=\{y \in \Omega: d(x, y)<r\}$ :

(1) the doubling condition (20) holds,

(2) the containment condition (19) holds,

(3) the Sobolev and Poincaré inequalities (21) and (23) hold with the given $\sigma$,

(4) the "accumulating sequence of Lipschitz cutoff functions" condition (27) holds for some $p>\max \left\{2 \sigma^{\prime}, 4\right\}$.

When restricted to the subunit balls $K(x, r)$ in (25) associated with a nonnegative semidefinite continuous quadratic form in $\Omega$, this theorem yields the following as a corollary since (27) holds automatically with $p=\infty$ in this case - see [11]. Since $L^{q}$ subellipticity in $\Omega$ is a local property, we may restrict attention to an arbitrary relatively compact subdomain $\Omega^{\prime}$, and in particular we may assume $Q$ is bounded if it is continuous.

Theorem 17. Suppose that $\mathcal{Q}(x, \xi)$ is a nonnegative semidefinite continuous quadratic form in a bounded open set $\Omega$, and suppose that the subunit metric $\delta(x, y)$ is finite on $\Omega \times \Omega$. Let the corresponding subunit balls $K(x, r)$ have upper dimension $Q^{*}$, and suppose that $\sigma>1$. Then $\mathcal{Q}(x, \xi)$ is $L^{q}$-subelliptic in $\Omega$ for $q>Q=\max \left\{Q^{*}, 2 \sigma^{\prime}\right\}$ provided that:

(1) the doubling condition $|K(x, 2 r)| \leq C|K(x, r)|$ holds for $0<r<\infty$,

(2) the containment condition (24) holds,

(3) the Sobolev and Poincaré inequalities (21) and (23) hold with $B(x, r)=$ $K(x, r)$ and the given $\sigma$.

Theorem 18. The statements of Theorems 12, 17, 20,23 and 24 in 11] also hold with the new definition of $L^{q}$-subelliptic in terms of $W_{\mathcal{Q}}$-weak solutions.

Proof. The proofs in 11] reduce matters to Theorems 16] and 17] above.

4.1. Proof of regularity. The proof of Theorem 16 proceeds exactly as in Chapter 3 of [1] using analogues for $W_{\mathcal{Q}}^{1,2}(\Omega)$ of the standard calculus for the classical space $W^{1,2}(\Omega)$, and upon replacing the vector-valued space $L^{2}(\Omega)$ (more precisely $\bigoplus_{k=1}^{n} L^{2}(\Omega)$ ) with the form-weighted vector-valued space $\mathcal{L}^{2}(\Omega, \mathcal{Q})$. Note that in [11] we made the hypothesis that the form $\mathcal{Q}$ is bounded. However, this was only used qualitatively in order to make sense of integrals involving the standard weak solutions used there, and is not needed here with our $W_{\mathcal{Q}}$ weak solutions. We now describe the needed changes. For $(u, \nabla u) \in \mathcal{W}_{\mathcal{Q}}^{1,2}(\Omega)$, define $u_{+}=\chi_{\{u>0\}} u$ and $u_{-}=\chi_{\{u<0\}} u$ so that $u_{ \pm}$is the composition of $t_{ \pm}=t \chi_{(0, \infty)}( \pm t)$ with $u$. Note that $t_{ \pm}$fails to be $C^{1}$ only at the origin. We now prove an analogue of Lemma 1 
for $\mathcal{W}_{\mathcal{Q}}^{1,2}(\Omega)$. A variant of the following lemma also appears in [10] for the calculus in $W_{\mathcal{Q}}^{1,2}(\Omega)$ defined using Cauchy sequences.

For $1 \leq p<\infty$ we define $\mathcal{L}^{p}(\Omega, \mathcal{Q})$ to consist of the measurable $\mathbb{R}^{n}$-valued functions $\mathbf{f}$ such that

$$
\|\mathbf{f}\|_{\mathcal{L}^{p}(\Omega, \mathcal{Q})}=\left(\int_{\Omega}\left(\mathbf{f}^{\prime} Q \mathbf{f}\right)^{\frac{p}{2}}\right)^{\frac{1}{p}}<\infty
$$

and if we identify $\mathbf{f}$ and $\mathbf{g}$ for which $\|\mathbf{f}-\mathbf{g}\|_{\mathcal{L}^{p}(\Omega, \mathcal{Q})}=0$, then $\mathcal{L}^{p}(\Omega, \mathcal{Q})$ is a normed linear space. From (9) we obtain

$$
\|\mathbf{f}\|_{\mathcal{L}^{p}(\Omega, \mathcal{Q})}=\left(\int_{\Omega}\left(\sum_{j=1}^{n}\left|f_{j}\right|^{2} \lambda_{j}\right)^{\frac{p}{2}}\right)^{\frac{1}{p}} \approx \sum_{j=1}^{n}\left\|f_{j}\right\|_{L^{p}\left(\lambda_{j}^{\frac{p}{2}}\right)}
$$

and the standard proof of completeness of $L^{p}(\mu)$, together with our proof of Theorem 4 above, now shows that $\mathcal{L}^{p}(\Omega, \mathcal{Q})$ is complete and hence a Banach space for $1 \leq p<\infty$.

Definition 19. We define $\mathcal{W}_{\mathcal{Q}}^{1, p}(\Omega)$ to be the closure of $\operatorname{Lip}_{\mathcal{Q}}(\Omega)$ in $L^{p}(\Omega) \times$ $\mathcal{L}^{p}(\Omega, \mathcal{Q})$, where $\operatorname{Lip}_{\mathcal{Q}}(\Omega)$ is embedded into the product space by sending $w$ to $(w, \nabla w)$.

Definition 20. Given $\alpha \in \mathbb{R}$, we define $\left(\mathcal{W}_{\mathcal{Q}}^{1,2}(\Omega)\right)_{\alpha}$ to be the completion in $\mathcal{W}_{\mathcal{Q}}^{1,2}(\Omega)$ of those Lipschitz functions $u$ in $\Omega$ such that $u-\alpha$ has compact support in $\Omega$.

Lemma 21. Suppose $(u, \nabla u) \in \mathcal{W}_{\mathcal{Q}}^{1,2}(\Omega)$ where $\Omega$ is bounded.

(1) If $\varphi \in \operatorname{Lip}_{\mathcal{Q}}(\Omega)$, then $(\varphi u, \varphi \nabla u+u \nabla \varphi) \in \mathcal{W}_{\mathcal{Q}}^{1,1}(\Omega)$.

(2) If $f \in C^{1}(\mathbb{R})$ with $f^{\prime} \in L^{\infty}(\mathbb{R})$, then $\left(f \circ u,\left(f^{\prime} \circ u\right) \nabla u\right) \in \mathcal{W}_{\mathcal{Q}}^{1,2}(\Omega)$. Also, if $u(x) \geq a>b$ and $f \in C^{1}(b, \infty)$ with $f^{\prime} \in L^{\infty}(b, \infty)$, then $\left(f \circ u,\left(f^{\prime} \circ u\right) \nabla u\right) \in \mathcal{W}_{\mathcal{Q}}^{1,2}(\Omega)$.

(3) Both $\left(u_{+}, \chi_{\{u>0\}} \nabla u\right)$ and $\left(u_{-}, \chi_{\{u<0\}} \nabla u\right)$ are in $\mathcal{W}_{\mathcal{Q}}^{1,2}(\Omega)$. Moreover, if $u \in\left(\mathcal{W}_{\mathcal{Q}}^{1,2}\right)_{\alpha}(\Omega)$ and $\alpha \geq 0$, then $\left(u_{+}, \chi_{\{u>0\}} \nabla u\right) \in\left(\mathcal{W}_{\mathcal{Q}}^{1,2}\right)_{\alpha}(\Omega)$ and $\left(u_{-}, \chi_{\{u<0\}} \nabla u\right) \in\left(\mathcal{W}_{\mathcal{Q}}^{1,2}\right)_{0}(\Omega)$ - the opposite holds if $\alpha<0$.

In the case that gradients are uniquely determined in $W_{\mathcal{Q}}^{1,2}(\Omega)$, we thus have

$$
\begin{aligned}
\nabla(\varphi u) & =\varphi \nabla u+u \nabla \varphi, \\
\nabla(f \circ u) & =\left(f^{\prime} \circ u\right) \nabla u, \\
\nabla u_{+} & =\chi_{\{u>0\}} \nabla u, \\
\nabla u_{-} & =\chi_{\{u<0\}} \nabla u,
\end{aligned}
$$

in $\mathcal{L}^{2}(\Omega, \mathcal{Q})$.

Proof. There is a sequence $\left\{u_{m}\right\} \subset \operatorname{Lip}_{\mathcal{Q}}(\Omega)$ such that $u_{m} \rightarrow u$ in $L^{2}(\Omega)$ and $\nabla u_{m} \rightarrow \nabla u$ in $\mathcal{L}^{2}(\Omega, \mathcal{Q})$ (we remind the reader that $\nabla u$ is not uniquely determined 
by $u$, but rather by the Cauchy sequence $\left.\left\{u_{m}\right\}\right)$. Then $\varphi u_{m} \in \operatorname{Lip}_{\mathcal{Q}}(\Omega)$, and suppressing dependence on $x$ we compute that

$$
\begin{aligned}
\int_{\Omega}\left|\varphi u_{m}-\varphi u\right| & \leq\|\varphi\|_{\infty}|\Omega|^{\frac{1}{2}}\left(\int_{\Omega}\left|u_{m}-u\right|^{2}\right)^{\frac{1}{2}} \rightarrow 0 \text { as } m \rightarrow \infty ; \\
\int_{\Omega} \mathcal{Q}\left(\nabla\left(\varphi u_{m}\right)-\{\varphi \nabla u+u \nabla \varphi\}\right)^{\frac{1}{2}} & =\int_{\Omega} \mathcal{Q}\left(\varphi\left\{\nabla u_{m}-\nabla u\right\}+\left\{u-u_{m}\right\} \nabla \varphi\right)^{\frac{1}{2}} \\
& \leq\|\varphi\|_{\infty}|\Omega|^{\frac{1}{2}} \int_{\Omega} \mathcal{Q}\left(\nabla u_{m}-\nabla u\right) \\
& +\left(\int_{\Omega} \mathcal{Q}(\nabla \varphi)\right)^{\frac{1}{2}}\left(\int_{\Omega}\left|u_{m}-u\right|^{2}\right)^{\frac{1}{2}}
\end{aligned}
$$

tends to 0 as $m \rightarrow \infty$, which proves assertion 1 .

Moreover, $f \circ u_{m} \in \operatorname{Lip}_{\mathcal{Q}}(\Omega)$ and

$$
\begin{aligned}
\int_{\Omega}\left|f\left(u_{m}\right)-f(u)\right|^{2} & \leq\left\|f^{\prime}\right\|_{\infty}^{2} \int_{\Omega}\left|u_{m}-u\right|^{2} \rightarrow 0 \quad \text { as } m \rightarrow \infty ; \\
\int_{\Omega} \mathcal{Q}\left(f^{\prime}\left(u_{m}\right) \nabla u_{m}-f^{\prime}(u) \nabla u\right) & \leq 2\left\|f^{\prime}\right\|_{\infty}^{2} \int_{\Omega} \mathcal{Q}\left(\nabla u_{m}-\nabla u\right) \\
& +2 \int_{\Omega}\left|f^{\prime}\left(u_{m}\right)-f^{\prime}(u)\right|^{2} \mathcal{Q}(\nabla u)
\end{aligned}
$$

also tends to 0 as $m \rightarrow \infty$ upon applying the dominated convergence theorem to the last integral using the continuity of $f^{\prime}$ and assuming, as we may by passing to a subsequence, that $u_{m} \rightarrow u$ a.e. in $\Omega$. This shows that $f \circ u_{m} \rightarrow f \circ u$ in $L^{2}(\Omega)$, and $\nabla\left(f \circ u_{m}\right)=\left(f^{\prime} \circ u_{m}\right) \nabla u_{m} \rightarrow\left(f^{\prime} \circ u\right) \nabla u$ in $\mathcal{L}^{2}(\Omega, \mathcal{Q})$, and completes the proof of the first statement in assertion 2. The second statement in the assertion follows upon applying the first statement to $\widetilde{f} \in C^{1}(\mathbb{R})$ with $\widetilde{f}^{\prime} \in L^{\infty}(\mathbb{R})$, where $\widetilde{f}$ agrees with $f$ on $\left(\frac{a+b}{2}, \infty\right)$.

Now suppose that $(u, \nabla u) \in \mathcal{W}_{\mathcal{Q}}^{1,2}(\Omega)$. For $\varepsilon>0$ define

$$
f_{\varepsilon}(u)=\left\{\begin{array}{ccc}
\sqrt{u^{2}+\varepsilon^{2}}-\varepsilon & \text { if } & u>0 \\
0 & \text { if } & u \leq 0
\end{array}\right.
$$

By assertion 2 we have using $f_{\varepsilon}^{\prime}(0)=0$,

$$
\nabla\left(f_{\varepsilon} \circ u\right)=\left(f_{\varepsilon}^{\prime} \circ u\right) \nabla u=\chi_{\{u>0\}} \frac{u}{\sqrt{u^{2}+\varepsilon^{2}}} \nabla u
$$

in $\mathcal{L}^{2}(\Omega, \mathcal{Q})$ for each $\varepsilon>0$. Letting $\varepsilon \rightarrow 0$ we obtain $f_{\varepsilon} \circ u \rightarrow u_{+}$in $L^{2}(\Omega)$ and $\nabla\left(f_{\varepsilon} \circ u\right) \rightarrow \chi_{\{u>0\}} \nabla u$ in $\mathcal{L}^{2}(\Omega, \mathcal{Q})$ by the monotone convergence theorem. Thus we obtain $\left(u_{+}, \chi_{\{u>0\}} \nabla u\right) \in \mathcal{W}_{\mathcal{Q}}^{1,2}(\Omega)$ since $\mathcal{W}_{\mathcal{Q}}^{1,2}(\Omega)$ is closed in $L^{2}(\Omega) \times \mathcal{L}^{2}(\Omega, \mathcal{Q})$. Similarly $\left(u_{-}, \chi_{\{u<0\}} \nabla u\right) \in \mathcal{W}_{\mathcal{Q}}^{1,2}(\Omega)$.

Now we turn to the final statement in assertion 3. Let $(u, \nabla u) \in\left(\mathcal{W}_{\mathcal{Q}}^{1,2}\right)_{\alpha}(\Omega)$. Suppose first that $\alpha=0$, and let $\left\{u_{k}\right\}_{k=1}^{\infty}$ be a sequence in $\operatorname{Lip}_{c}(\Omega)$, the space of compactly supported Lipschitz functions in $\Omega$, that converges to $(u, \nabla u) \in$ $\left(\mathcal{W}_{\mathcal{Q}}^{1,2}\right)_{0}(\Omega)$. Since $f_{\varepsilon}(0)=0$, we see that $f_{\varepsilon} \circ u_{k} \in \operatorname{Lip}_{c}(\Omega)$ and so

$$
f_{\varepsilon} \circ u=\lim _{k \rightarrow \infty} f_{\varepsilon} \circ u_{k} \in\left(\mathcal{W}_{\mathcal{Q}}^{1,2}\right)_{0}(\Omega), \quad 0<\varepsilon<1 .
$$


It now follows that

$$
\left(u_{+}, \chi_{\{u>0\}} \nabla u\right)=\lim _{\varepsilon \rightarrow 0} f_{\varepsilon} \circ u \in\left(\mathcal{W}_{\mathcal{Q}}^{1,2}\right)_{0}(\Omega) .
$$

If $\alpha<0$ and $\left\{u_{k}\right\}_{k=1}^{\infty}$ is a sequence in $\operatorname{Lip}_{c}(\Omega)$ such that $u_{k}+\alpha$ converges to $(u, \nabla u)$, then $\left(u_{k}+\alpha\right)_{+} \in \operatorname{Lip}_{c}(\Omega)$ and it follows that $\left(u_{+}, \chi_{\{u>0\}} \nabla u\right) \in\left(\mathcal{W}_{\mathcal{Q}}^{1,2}\right)_{0}(\Omega)$.

Now suppose that $\alpha>0$. We must left translate the functions $f_{\varepsilon}$ to satisfy $f_{\varepsilon}(\alpha)=\alpha$. Thus we define

$$
f_{\varepsilon}^{\alpha}(u)=f_{\varepsilon}(u+t(\alpha, \varepsilon)), \quad u \in \mathbb{R},
$$

where $t(\alpha, \varepsilon)$ is the unique positive number satisfying

$$
\sqrt{(\alpha+t(\alpha, \varepsilon))^{2}+\varepsilon^{2}}-\varepsilon=f_{\varepsilon}(\alpha+t(\alpha, \varepsilon))=\alpha .
$$

If $\left\{u_{k}-\alpha\right\}_{k=1}^{\infty}$ is a sequence in $\operatorname{Lip}_{c}(\Omega)$ such that $\left\{u_{k}\right\}_{k=1}^{\infty}$ converges to $(u, \nabla u) \in$ $\left(\mathcal{W}_{\mathcal{Q}}^{1,2}\right)_{\alpha}(\Omega)$, then since $f_{\varepsilon}^{\alpha}(\alpha)=\alpha$, we see that

$$
f_{\varepsilon}^{\alpha} \circ u=\lim _{k \rightarrow \infty} f_{\varepsilon}^{\alpha} \circ u_{k} \in\left(\mathcal{W}_{\mathcal{Q}}^{1,2}\right)_{\alpha}(\Omega), \quad 0<\varepsilon<1 .
$$

Since $t(\alpha, \varepsilon) \rightarrow 0$ as $\varepsilon \rightarrow 0$, we also have that $\lim _{\varepsilon \rightarrow 0} f_{\varepsilon}^{\alpha}(u)=u_{+}$in $L^{2}(\Omega)$ and $\nabla\left(f_{\varepsilon}^{\alpha} \circ u\right) \rightarrow \chi_{\{u>0\}} \nabla u$ in $\mathcal{L}^{2}(\Omega, \mathcal{Q})$ by the dominated convergence theorem. Thus it follows that

$$
\left(u_{+}, \chi_{\{u>0\}} \nabla u\right)=\lim _{\varepsilon \rightarrow 0} f_{\varepsilon}^{\alpha} \circ u \in\left(\mathcal{W}_{\mathcal{Q}}^{1,2}\right)_{\alpha}(\Omega)
$$

since $\left(\mathcal{W}_{\mathcal{Q}}^{1,2}\right)_{\alpha}(\Omega)$ is a closed set in $L^{2}(\Omega) \times \mathcal{L}^{2}(\Omega, \mathcal{Q})$.

Similarly $\left(u_{-}, \chi_{\{u<0\}} \nabla u\right) \in\left(\mathcal{W}_{\mathcal{Q}}^{1,2}\right)_{\alpha}(\Omega)$ for $\alpha<0$ and $\left(u_{-}, \chi_{\{u<0\}} \nabla u\right) \in$ $\left(\mathcal{W}_{\mathcal{Q}}^{1,2}\right)_{0}(\Omega)$ for $\alpha \geq 0$, and this completes the proof of Lemma 21.

We will also need an extension of part 2 of Lemma 21 to include $f \in C_{p w}^{1}(\mathbb{R})$ with $f^{\prime} \in L^{\infty}(\mathbb{R})$, where $C_{p w}^{1}(\mathbb{R})$ is the space of piecewise continuously differentiable functions on $\mathbb{R}$, i.e. $f$ is continuous, $f^{\prime}$ has at most finitely many discontinuities $\mathcal{D}_{f^{\prime}}$ and $f^{\prime}$ has two-handed limits at each point in $\mathcal{D}_{f^{\prime}}$. The immediate difficulty is that $f^{\prime} \circ u$ is undefined on the set where $u$ takes values in $\mathcal{D}_{f^{\prime}}$, which can lead to problems if this set has positive measure. This motivates the following definition.

Definition 22. We say that an element $(u, \nabla u) \in \mathcal{W}_{\mathcal{Q}}^{1,2}(\Omega)$ is regular if

$$
\left\|\chi_{\{u=\alpha\}} \nabla u\right\|_{\mathcal{L}^{2}(\Omega, \mathcal{Q})}=0 \text { for all } \alpha \in \mathbb{R} .
$$

One way out of the difficulty mentioned above is to simply assume that $\mathcal{Q}(x, \xi) \approx$ $\mathcal{X}(x, \xi)$, where $\mathcal{X}=\left\{X_{j}\right\}_{j=1}^{m}$ is a collection of $H_{\text {div }}^{1,2}(\Omega)$ vector fields, since then the elements in $W_{\mathcal{Q}}^{1,2}(\Omega)$ are necessarily regular in the sense of (35), as we now show.

Corollary 23. Suppose that $\mathcal{Q}(x, \xi)$ is comparable to the quadratic form $\mathcal{X}(x, \xi)$ associated to a collection $\mathcal{X}=\left\{X_{j}\right\}_{j=1}^{m}$ of $H_{\mathrm{div}}^{1,2}(\Omega)$ vector fields in a bounded open set $\Omega$. Then $\nabla u$ is uniquely determined in $\mathcal{L}^{2}(\Omega, \mathcal{Q})$ and every $u \in W_{\mathcal{Q}}^{1,2}(\Omega)$ is regular, i.e. (35) holds. 
Proof. As observed earlier, $W_{\mathcal{Q}}^{1,2}(\Omega)$ is embedded in $H_{\mathcal{X}}^{1,2}(\Omega)$ under these conditions, and it follows that $\mathcal{X} u$ is uniquely determined in $L^{2}(\Omega)$ if $u \in W_{\mathcal{Q}}^{1,2}(\Omega)$, hence by the comparability of $\mathcal{Q}$ and $\mathcal{X}$, that $\nabla u$ is uniquely determined in $\mathcal{L}^{2}(\Omega, \mathcal{Q})$. Since $u=u_{+}+u_{-}$in $L^{2}(\Omega)$, uniqueness of gradients shows that $\nabla u=\nabla u_{+}+$ $\nabla u_{-}=\chi_{\{u \neq 0\}} \nabla u$ in $\mathcal{L}^{2}(\Omega, \mathcal{Q})$. Thus $\nabla u$ vanishes on the set where $u$ is zero, i.e. $\left\|\chi_{\{u=0\}} \nabla u\right\|_{\mathcal{L}^{2}(\Omega, \mathcal{Q})}=0$. Applying this argument to $u-\alpha$ for constants $\alpha \in \mathbb{R}$ yields (35).

However, there is another way around this difficulty that does not require we suppose the weak solution $u$ is regular. Instead, we use the following proposition that shows for every $(u, \mathbf{v}) \in \mathcal{W}_{\mathcal{Q}}^{1,2}(\Omega)$ there is a natural choice of $\mathbf{w} \in \mathcal{L}^{2}(\Omega, \mathcal{Q})$ such that $(u, \mathbf{w}) \in \mathcal{W}_{\mathcal{Q}}^{1,2}(\Omega)$ and $(u, \mathbf{w})$ satisfies (35).

Proposition 24. Suppose that $(u, \nabla u) \in \mathcal{W}_{\mathcal{Q}}^{1,2}(\Omega)$ (respectively $\left.\left(\mathcal{W}_{\mathcal{Q}}^{1,2}\right)_{0}(\Omega)\right)$, where $\Omega$ is bounded, and let

$$
\mathcal{R}_{u}=\{\alpha \in \mathbb{R}: u=\alpha \text { on a set of positive measure }\} .
$$

With $\nabla_{\text {reg }} u=\chi_{\left\{x \in \Omega: u(x) \notin \mathcal{R}_{u}\right\}} \nabla u$ we have $\left(u, \nabla_{\text {reg }} u\right) \in \mathcal{W}_{\mathcal{Q}}^{1,2}(\Omega)$ (respectively $\left.\left(\mathcal{W}_{\mathcal{Q}}^{1,2}\right)_{0}(\Omega)\right)$ and $\left(u, \nabla_{\text {reg }} u\right)$ satisfies (35).

We caution the reader that our definition of $\nabla_{\text {reg }} u$ depends on both $u$ and $\nabla u$. We do not know if $\nabla_{\text {reg }} u$ is uniquely determined by $u$.

If in addition the Sobolev inequality (21) and the Poincaré inequality (23) hold, we may of course restrict $\nabla u$ to $\nabla_{r e g} u$ in the right side of both inequalities. Note that $\nabla_{\text {reg }} u$ is essentially a smallest of the gradients that when paired with $u$ belong to $\mathcal{W}_{\mathcal{Q}}^{1,2}(\Omega)$.

Proof. The set $\mathcal{R}_{u}$ is at most countable, so we can enumerate it as $\left\{\alpha_{j}\right\}_{j=1}^{\infty}$ (the case when $\mathcal{R}_{u}$ is finite is easier). If $\alpha_{1}=0$, then assertion 3 of Lemma 21 shows that $\left(u, \chi_{\{u \neq 0\}} \nabla u\right)=\left(u_{+}, \chi_{\{u>0\}} \nabla u\right)+\left(u_{-}, \chi_{\{u<0\}} \nabla u\right) \in \mathcal{W}_{\mathcal{Q}}^{1,2}(\Omega)$. In general, as $\left(u-\alpha_{1}, \nabla u\right) \in \mathcal{W}_{\mathcal{Q}}^{1,2}(\Omega)$,

$$
\begin{aligned}
\left(u-\alpha_{1}, \chi_{\left\{u \neq \alpha_{1}\right\}} \nabla u\right)= & \left(\left(u-\alpha_{1}\right)_{+}, \chi_{\left\{u>\alpha_{1}\right\}} \nabla u\right) \\
& +\left(\left(u-\alpha_{1}\right)_{-}, \chi_{\left\{u<\alpha_{1}\right\}} \nabla u\right) \in \mathcal{W}_{\mathcal{Q}}^{1,2}(\Omega),
\end{aligned}
$$

so that $\left(u, \chi_{\left\{u \neq \alpha_{1}\right\}} \nabla u\right)=\left(u-\alpha_{1}, \chi_{\left\{u \neq \alpha_{1}\right\}} \nabla u\right)+\left(\alpha_{1}, 0\right) \in \mathcal{W}_{\mathcal{Q}}^{1,2}(\Omega)$, and by induction,

$$
\left(u, \chi_{\left\{u \neq \alpha_{1}, \ldots \alpha_{k}\right\}} \nabla u\right) \in \mathcal{W}_{\mathcal{Q}}^{1,2}(\Omega), \quad 1 \leq k<\infty .
$$

Now let $k \rightarrow \infty$ and note that $\chi_{\left\{u \neq \alpha_{1}, \ldots \alpha_{k}\right\}} \nabla u \rightarrow \chi_{\left\{x \in \Omega: u(x) \notin \mathcal{R}_{u}\right\}} \nabla u=\nabla_{\text {reg }} u$ pointwise. We then obtain that $\chi_{\left\{u \neq \alpha_{1}, \ldots \alpha_{k}\right\}} \nabla u \rightarrow \nabla_{\text {reg }} u$ in $\mathcal{L}^{2}(\Omega, \mathcal{Q})$ by the dominated convergence theorem. Thus $\left(u, \nabla_{\text {reg }} u\right) \in \mathcal{W}_{\mathcal{Q}}^{1,2}(\Omega)$ since $\mathcal{W}_{\mathcal{Q}}^{1,2}(\Omega)$ is closed, and we trivially have that $\left(u, \nabla_{\text {reg }} u\right)$ satisfies (35).

The case when $(u, \nabla u) \in\left(\mathcal{W}_{\mathcal{Q}}^{1,2}\right)_{0}(\Omega)$ is handled in the same way using the final statement in assertion 3 of Lemma 21. Indeed, for $\alpha_{1} \geq 0$, we have $\left(u-\alpha_{1}, \nabla u\right) \in$ 
$\left(\mathcal{W}_{\mathcal{Q}}^{1,2}\right)_{-\alpha_{1}}(\Omega)$ and then

$$
\begin{aligned}
& \left(\left(u-\alpha_{1}\right)_{+}, \chi_{\left\{u>\alpha_{1}\right\}} \nabla u\right) \in\left(\mathcal{W}_{\mathcal{Q}}^{1,2}\right)_{0}(\Omega), \\
& \left(\left(u-\alpha_{1}\right)_{-}, \chi_{\left\{u<\alpha_{1}\right\}} \nabla u\right) \in\left(\mathcal{W}_{\mathcal{Q}}^{1,2}\right)_{-\alpha_{1}}(\Omega),
\end{aligned}
$$

and so

$$
\begin{aligned}
\left(u-\alpha_{1}, \chi_{\left\{u \neq \alpha_{1}\right\}} \nabla u\right) & =\left(\left(u-\alpha_{1}\right)_{+}, \nabla\left(u-\alpha_{1}\right)_{+}\right)+\left(\left(u-\alpha_{1}\right)_{-}, \nabla\left(u-\alpha_{1}\right)_{-}\right) \\
& \in\left(\mathcal{W}_{\mathcal{Q}}^{1,2}\right)_{0}(\Omega)+\left(\mathcal{W}_{\mathcal{Q}}^{1,2}\right)_{-\alpha_{1}}(\Omega)=\left(\mathcal{W}_{\mathcal{Q}}^{1,2}\right)_{-\alpha_{1}}(\Omega) .
\end{aligned}
$$

Thus

$$
\begin{aligned}
\left(u, \chi_{\left\{u \neq \alpha_{1}\right\}} \nabla u\right) & =\left(u-\alpha_{1}, \chi_{\left\{u \neq \alpha_{1}\right\}} \nabla u\right)+\left(\alpha_{1}, 0\right) \\
& \in\left(\mathcal{W}_{\mathcal{Q}}^{1,2}\right)_{-\alpha_{1}}(\Omega)+\left(\mathcal{W}_{\mathcal{Q}}^{1,2}\right)_{\alpha_{1}}(\Omega)=\left(\mathcal{W}_{\mathcal{Q}}^{1,2}\right)_{0}(\Omega) .
\end{aligned}
$$

The argument is similar for $\alpha_{1}<0$, and we can now proceed by induction as before.

Corollary 25. Suppose $(u, \nabla u) \in \mathcal{W}_{\mathcal{Q}}^{1,2}(\Omega)$ with $\Omega$ bounded and that either $f \in$ $C_{p w}^{1}(\mathbb{R})$ with $f^{\prime} \in L^{\infty}(\mathbb{R})$, or $u(x) \geq a>b$ and $f \in C_{p w}^{1}(b, \infty)$ with $f^{\prime} \in$ $L^{\infty}(b, \infty)$. Then

$$
\left(f \circ u,\left(f^{\prime} \circ u\right) \nabla_{r e g} u\right) \in \mathcal{W}_{\mathcal{Q}}^{1,2}(\Omega) .
$$

Remark 26. In the situation of Corollary 23, we thus have $\nabla u=\nabla_{\text {reg }} u$ and $\nabla(f \circ u)=\left(f^{\prime} \circ u\right) \nabla u$.

Proof. Choose a sequence $\left\{g_{k}\right\}_{k=1}^{\infty}$ in $C^{1}(\mathbb{R})$ with uniformly bounded derivatives that converges uniformly to $f$ on $\mathbb{R}$ and such that $g_{k}^{\prime}$ converges pointwise and boundedly to $f^{\prime}$ on the set where $f^{\prime}$ is continuous. By Proposition 24. $\left(u, \nabla_{\text {reg }} u\right) \in$ $\mathcal{W}_{\mathcal{Q}}^{1,2}(\Omega)$, and from assertion 2 of Lemma 21 applied to $\left(u, \nabla_{\text {reg }} u\right)$ we obtain that

$$
\left(g_{k} \circ u, g_{k}^{\prime}(u) \nabla_{r e g} u\right) \in \mathcal{W}_{\mathcal{Q}}^{1,2}(\Omega), \quad 1 \leq k<\infty .
$$

Now we let $k \rightarrow \infty$ and note that

$$
g_{k}^{\prime}(u) \nabla_{r e g} u \rightarrow f^{\prime}(u) \nabla_{r e g} u
$$

pointwise a.e. upon recalling that if $u$ is constant on a set of positive measure, then $\nabla_{\text {reg }} u$ is zero on that set. Again, it follows from the dominated convergence theorem that $g_{k}^{\prime}(u) \nabla_{r e g} u \rightarrow f^{\prime}(u) \nabla_{r e g} u$ in $\mathcal{L}^{2}(\Omega, \mathcal{Q})$. Since we also have $g_{k} \circ u \rightarrow f(u)$ in $L^{2}(\Omega)$, we conclude that $\left(f(u), f^{\prime}(u) \nabla_{r e g} u\right)$ is in $\mathcal{W}_{\mathcal{Q}}^{1,2}(\Omega)$.

The following corollary is needed explicitly to prove the analogue of (113) in chapter 3 of [11] - see the discussion below. In fact, as we indicate in more detail below, this corollary together with Lemma 21, Proposition 24 and Corollary 25 suffice for all the calculus needed in the proof of Theorem 8 given in chapter 3 of [11.

Corollary 27. Suppose $\Omega$ is bounded and that $(u, \nabla u),(v, \nabla v) \in \mathcal{W}_{\mathcal{Q}}^{1,2}(\Omega)$. Then

$$
(u v, u \nabla v+v \nabla u) \in \mathcal{W}_{\mathcal{Q}}^{1,1}(\Omega) .
$$


Proof. Let $\left\{v_{m}\right\} \subset \operatorname{Lip}_{\mathcal{Q}}(\Omega)$ satisfy $v_{m} \rightarrow v$ in $L^{2}(\Omega)$ and $\nabla v_{m} \rightarrow \nabla v$ in $\mathcal{L}^{2}(\Omega, \mathcal{Q})$. Then by part 1 of Lemma 21 with $\varphi=v_{m}$ we have

$$
\left(u v_{m}, u \nabla v_{m}+v_{m} \nabla u\right) \in \mathcal{W}_{\mathcal{Q}}^{1,1}(\Omega) .
$$

Now we let $m \rightarrow \infty$ and use

$$
\left\|u v_{m}-u v\right\|_{L^{1}(\Omega)} \leq\|u\|_{L^{2}(\Omega)}\left\|v_{m}-v\right\|_{L^{2}(\Omega)}
$$

and

$$
\begin{aligned}
& \left\|\left(u \nabla v_{m}+v_{m} \nabla u\right)-(u \nabla v+v \nabla u)\right\|_{\mathcal{L}^{1}(\Omega, \mathcal{Q})} \\
& \leq \int_{\Omega}|u|\left|\mathcal{Q}\left(\nabla v_{m}-\nabla v\right)\right|^{\frac{1}{2}}+\int_{\Omega}\left|v_{m}-v\right||\mathcal{Q}(\nabla u)|^{\frac{1}{2}} \\
& \leq\|u\|_{L^{2}(\Omega)}\left\|\nabla v_{m}-\nabla v\right\|_{\mathcal{L}^{2}(\Omega, \mathcal{Q})}+\left\|v_{m}-v\right\|_{L^{2}(\Omega)}\|\nabla u\|_{\mathcal{L}^{2}(\Omega, \mathcal{Q})}
\end{aligned}
$$

to obtain $(u v, u \nabla v+v \nabla u) \in \mathcal{W}_{\mathcal{Q}}^{1,1}(\Omega)$.

We are indebted to S. Rodney for pointing out to us his treatment of products of Cauchy sequences in [10], which motivated the definition of products in $W_{\mathcal{Q}}^{1,2}(\Omega)$ that is used in the second bullet item below.

4.1.1. Adapting the classical proof. We end this subsection with some remarks on adapting the proof in chapter 3 of [11] to the situation at hand here.

In the special case that $\mathcal{X} \in H^{1,2 \sigma^{\prime}}(\Omega)$ and (16) holds for some $\sigma \geq 1$, then Proposition 29 below shows that $W_{\mathcal{X}}^{1, p}(\Omega)=H_{\mathcal{X}}^{1, p}(\Omega)$ for $1 \leq p<2 \sigma^{\prime}$. Thus for $u, v \in H_{\mathcal{X}}^{1,2}(\Omega)$, Corollary 27 yields $u v \in H_{\mathcal{X}}^{1,1}(\Omega)$. As a result we obtain the following.

Claim 28. Suppose $\mathcal{X} \in H^{1,2 \sigma^{\prime}}(\Omega), \sigma \geq 1$, and that $\Omega$ is bounded. If $u, v, u v \in$ $H_{\mathcal{X}}^{1,2}(\Omega)$ and if $u \in L^{\infty}(\Omega)$, then $v \nabla u \in \mathcal{L}^{2}(\Omega, \mathcal{X})$.

Indeed, the above corollary yields $\nabla(u v)=u \nabla v+v \nabla u$ in $\mathcal{L}^{1}(\Omega, \mathcal{X})$. By assumption both $\nabla v$ and $\nabla(u v)$ are in $\mathcal{L}^{2}(\Omega, \mathcal{X})$ and $u$ is bounded, so we conclude that both $\nabla(u v)$ and $u \nabla v$ are in $\mathcal{L}^{2}(\Omega, \mathcal{X})$; hence $v \nabla u \in \mathcal{L}^{2}(\Omega, \mathcal{X})$.

We can now obtain the required analogue of (113) on page 56 of [11 in this case. Using the notation of [11 we recall that (113) asserts $w h^{\prime \prime}(u) \nabla u \in L^{2}(\Omega)$ if $u \in W^{1,2}(\Omega)$ and $w$ is in the test function space (see (110) in [11]),

$$
\mathcal{M}[u ; h]=\left\{w \in W_{0}^{1,2}(\Omega): w \geq 0 \text { and } h^{\prime}(u) w \in W_{0}^{1,2}(\Omega)\right\},
$$

where $h$ is admissible for $u$; i.e., there is an interval $I$ containing the range of $u$ such that $h \in C^{1}(I) \cap C_{p w}^{2}(I)$ is positive and monotone on $I$ and

$$
\left|h^{\prime}(t)\right|,\left|h^{\prime \prime}(t)\right|,\left|t h^{\prime \prime}(t)\right| \leq C, \quad t \in I .
$$

Now suppose that $u \in H_{\mathcal{X}}^{1,2}(\Omega)$ and that $w$ is in the analogous test function space

$$
\mathcal{M}_{\mathcal{X}}[u ; h]=\left\{w \in\left(H_{\mathcal{X}}^{1,2}\right)_{0}(\Omega): w \geq 0 \text { and } h^{\prime}(u) w \in\left(H_{\mathcal{X}}^{1,2}\right)_{0}(\Omega)\right\},
$$

where $h$ is admissible for $u$. We first observe that

$$
h^{\prime} \circ u \in H_{\mathcal{X}}^{1,2}(\Omega) \text { with } \nabla\left(h^{\prime} \circ u\right)=h^{\prime \prime}(u) \nabla u,
$$

and Corollary 25 shows that $\left(h^{\prime} \circ u,\left(h^{\prime \prime} \circ u\right) \nabla_{\text {reg }} u\right) \in \mathcal{W}_{\mathcal{X}}^{1,2}(\Omega)$. Since gradients are unique in $H_{\mathcal{X}}^{1,2}=\mathcal{W}_{\mathcal{X}}^{1,2}(\Omega)$, we have $\nabla_{\text {reg }} u=\nabla u$, which completes the proof 
of (36). If we now apply the claim above with $u, v$ replaced by $h^{\prime} \circ u, w$ we obtain that $w h^{\prime \prime}(u) \nabla u=w \nabla\left(h^{\prime} \circ u\right) \in \mathcal{L}^{2}(\Omega, \mathcal{X})$, the required analogue of (113).

In the general case of a quadratic form $\mathcal{Q}$, we still obtain the above conclusions, but we need to take care in defining the composition $h^{\prime} \circ u$, as well as in defining what we mean by the product of two elements $(u, \nabla u)$ and $(v, \nabla v)$ in $\mathcal{W}_{\mathcal{Q}}^{1,2}(\Omega)$. We define $h^{\prime} \circ u$ to be the element $\left(h^{\prime}(u), h^{\prime \prime}(u) \nabla_{r e g} u\right) \in \mathcal{W}_{\mathcal{Q}}^{1,2}(\Omega)$ given by Corollary 25 i.e., we take $\nabla h^{\prime}(u)$ to be the element $h^{\prime \prime}(u) \nabla_{\text {reg }} u$ in $\mathcal{L}^{2}(\Omega, \mathcal{Q})$. Motivated by the approach using Cauchy sequences in [10, we define the product of $(u, \nabla u)$ and $(v, \nabla v)$ to be the pair $(u v, u \nabla v+v \nabla u)$, which lies in $\mathcal{W}_{\mathcal{Q}}^{1,1}(\Omega)$ by Corollary 27 If in the claim above we replace $\mathcal{X}$ by $\mathcal{Q}$ and the assumption $u v \in H_{\mathcal{X}}^{1,2}(\Omega)$ by $(u v, u \nabla v+v \nabla u) \in \mathcal{W}_{\mathcal{Q}}^{1,2}(\Omega)$, we again conclude by taking differences that $v \nabla u \in$ $\mathcal{L}^{2}(\Omega, \mathcal{Q})$. We now define the space of test functions $\mathcal{M}_{\mathcal{Q}}[u ; h]$ by

$$
\mathcal{M}_{\mathcal{Q}}[u ; h]=\left\{w \in\left(W_{\mathcal{Q}}^{1,2}\right)_{0}(\Omega): w \geq 0 \text { and } h^{\prime}(u) w \in\left(W_{\mathcal{Q}}^{1,2}\right)_{0}(\Omega)\right\}
$$

with the following two understandings:

- $h^{\prime}(u)$ refers to the pair $\left(h^{\prime}(u), h^{\prime \prime}(u) \nabla_{r e g} u\right) \in \mathcal{W}_{\mathcal{Q}}^{1,2}(\Omega)$, and

- if $\mathrm{U}, \mathrm{V} \in W_{\mathcal{Q}}^{1,2}$ are represented by the pairs $(u, \nabla u),(v, \nabla v) \in \mathcal{W}_{\mathcal{Q}}^{1,2}(\Omega)$, then the product UV is represented by the pair $(u v, u \nabla v+v \nabla u) \in \mathcal{W}_{\mathcal{Q}}^{1,1}(\Omega)$.

The appropriate analogue of (113) now holds here as well. Since the compositions $h \circ u$ appearing in [11] satisfy the hypotheses of assertion 2 of Lemma 21, the proof in [11] now carries over to prove Theorem [16 here if we simply interpret $h^{\prime \prime}(u)$ everywhere as $\chi_{\left\{x \in \Omega: u(x) \notin \mathcal{R}_{u}\right\}} h^{\prime \prime}(u)$, where $\mathcal{R}_{u}$ is the set of values $u$ takes on with positive measure as in Proposition 24.

More precisely, we have the following analogue of (112) in [11:

$$
\begin{aligned}
-\int \nabla w B \nabla(h \circ u)=-\int \nabla & {\left[w h^{\prime}(u)\right] B \nabla u } \\
& +\int w \chi_{\left\{x \in \Omega: u(x) \notin \mathcal{R}_{u}\right\}} h^{\prime \prime}(u)[\nabla u] B[\nabla u] .
\end{aligned}
$$

To see this we use $\nabla(h \circ u)=h^{\prime}(u) \nabla u$ from assertion 2 of Lemma 21 together with the two bullet items above.

Then to obtain the analogue of (114) with $\chi_{\left\{x \in \Omega: u(x) \notin \mathcal{R}_{u}\right\}} h^{\prime \prime}(u)$ in place of $h^{\prime \prime}(u)$, we use

$$
\int_{\Omega} \varphi T v=-\int_{\Omega} v T^{\prime} \varphi
$$

for $v \in W_{\mathcal{Q}}^{1,2}(\Omega), \varphi \in \operatorname{Lip}_{c}(\Omega)$ and $T$ subunit - this in turn is true by approximation of $v$ by Lipschitz functions using Remark 12.

Then (115) uses only (111) and $\nabla(h \circ u)=h^{\prime}(u) \nabla u$, and (116) follows directly from (111), (114) and (115). This shows that with $\widetilde{u}=h \circ u$ we have the following analogue of (117) in [11]:

$$
\widetilde{L} \widetilde{u}=\widetilde{f}+\mathbf{T}^{\prime} \widetilde{\mathbf{g}}+\chi_{\left\{x \in \Omega: u(x) \notin \mathcal{R}_{u}\right\}} h^{\prime \prime}(u)[\nabla u] B[\nabla u]+\Phi
$$

in the $\mathcal{M}_{\mathcal{Q}}[u ; h]$-weak sense, where $\Phi=\chi_{\left\{x \in \Omega: u(x) \notin \mathcal{R}_{u}\right\}} h^{\prime \prime}(u)\{(\mathbf{T} u) \mathbf{g}-(\mathbf{S} u)(\mathbf{G} u)\}$ and $\widetilde{L}, \widetilde{f}$ and $\widetilde{\mathbf{g}}$ are as on page 58 of [11]. The meaning of equation (37) in the $\mathcal{M}_{\mathcal{Q}}[u ; h]$-weak sense is that if we multiply both sides by $w \in \mathcal{M}_{\mathcal{Q}}[u ; h]$, and then integrate by parts formally in the appropriate integrals, the resulting integrals are 
absolutely convergent and equality of the two sides holds. Finally, in Subsection 3.2 on p. 81 of [11] we revert to using equation (99) there instead of (116) or (117) there, and the reader can verify that the argument in Subsection 3.2 also carries over with $\chi_{\left\{x \in \Omega: u(x) \notin \mathcal{R}_{u}\right\}} h^{\prime \prime}(u)$ in place of $h^{\prime \prime}(u)$. We should also point out that the remaining arguments in chapter 3 of [11] do not use any calculus for the functions $u_{+}=\max \{u, 0\}$ and $u_{-}=\min \{u, 0\}$, only pointwise estimates.

Recall from Corollary 23 that when the quadratic form $\mathcal{Q}(x, \xi)$ is comparable to the form $\mathcal{X}(x, \xi)$ associated to a collection $\mathcal{X}$ of $H_{\text {div }}^{1,2}(\Omega)$ vector fields, the gradient $\nabla u$ of $u \in \mathrm{W}_{\mathcal{Q}}^{1,2}(\Omega)$ is uniquely determined as an element in the form-weighted space $\mathcal{L}^{2}(\Omega, \mathcal{Q})$, and moreover elements in $\mathrm{W}_{\mathcal{Q}}^{1,2}(\Omega)$ are regular, i.e. (35) holds. In this case, the proof in Chapter 3 of 11 applies verbatim to $W_{\mathcal{Q}}$-weak solutions $u \in L^{2}(\Omega)$ with $\nabla u \in \mathcal{L}^{2}(\Omega, \mathcal{Q})$ provided $(u, \nabla u) \in \mathcal{W}_{\mathcal{Q}}^{1,2}(\Omega)$.

\section{Equality of Degenerate Sobolev spaces}

We prove equality of degenerate $H$ and $W$ Sobolev spaces for $H^{1,2}(\Omega)$ vector fields $\mathcal{X}$ in three situations:

(1) when the vector fields $\mathcal{X}$ are in $H^{1,2 \sigma^{\prime}}(\Omega)$ and satisfy the Sobolev inequality (16) for the same $\sigma \geq 1$;

(2) when the vector fields $\mathcal{X}$ are in $H^{1,2}(\Omega)$, are comparably Lipschitz off their common zero set $Z$ (see (41) below), and Lipschitz at $Z$, i.e. $\left|\mathbf{v}_{j}(x)\right| \leq$ Cdist $(x, Z)$;

(3) when the dimension $n=1$ and the vector fields $\mathcal{X}$ are in $H^{1,2}(\Omega)$.

For convenience we will state and prove our results for $1 \leq p<\infty$ when appropriate. We begin by considering the first situation in the following subsection.

5.1. The Sobolev inequality. The following proposition is proved in 3 ] and [4] in the case when the vector fields $\mathcal{X}=\left\{X_{1}, \ldots, X_{m}\right\}$ are Lipschitz continuous in $\Omega$. Recall that

$$
H^{1, p}(\Omega)=\left\{v \in L^{p}(\Omega): \nabla v \in L^{p}(\Omega)\right\},
$$

and consider the following $L^{p}-L^{q}$ analogue of (16):

$$
\left\{\int_{\omega}|w|^{q}\right\}^{\frac{1}{q}} \leq C_{p, q, \omega}\left\{\int_{\omega}|w|^{p}+\int_{\omega}|\mathcal{X} w|^{p}\right\}^{\frac{1}{p}},
$$

for all opens sets $\omega \Subset \Omega$ and for all $w \in \operatorname{Lip}_{c}(\omega)$. Note that (38) typically holds in a stronger form in the presence of a subrepresentation inequality in a homogeneous space with $\frac{1}{q}=\frac{1}{p}-\frac{1}{D}$, where $D$ is the doubling exponent for the metric balls; see e.g. Proposition 74 in [11. Inequalities (16) and (38) coincide when $q=2 \sigma, p=2$ and $D=2 \sigma^{\prime}$. Below we give an example of vector fields $\mathcal{X}$ satisfying the hypotheses of Proposition 29, but with $\mathcal{X}(x, \xi)$ not comparable to any quadratic form arising from Lipschitz vector fields.

Proposition 29. Suppose that $\mathcal{X}=\left\{X_{1}, \ldots, X_{m}\right\}$ is a collection of $H^{1, D}(\Omega)$ vector fields in $\Omega$ satisfying (38) with $\frac{1}{q}=\frac{1}{p}-\frac{1}{D}$ for some $1 \leq p<D$. Then $W_{\mathcal{X}}^{1, p}(\Omega)=$ $H_{\mathcal{X}}^{1, p}(\Omega)$; moreover, $J_{\varepsilon} * \varphi f \rightarrow \varphi f$ in $H_{\mathcal{X}}^{1, p}(\Omega)$ as $\varepsilon \rightarrow 0$ for every $f \in H_{\mathcal{X}}^{1, p}(\Omega)$, $\varphi \in C_{c}^{\infty}(\Omega)$ and any smooth compactly supported Euclidean approximate identity $\left\{J_{\varepsilon}\right\}_{0<\varepsilon<1}$. 
Remark 30. In general, however, convolution of elements in $H_{\mathcal{X}}^{1, p}(\Omega)$ is not sufficient for proving local density of $\operatorname{Lip}(\Omega)$ when $\mathcal{X}$ is not Lipschitz. See the subsection on examples below.

Proof. The proof is a straightforward modification of that of Theorem A.2 in [4, where our spaces $W_{\mathcal{X}}^{1, p}(\Omega)$ and $H_{\mathcal{X}}^{1, p}(\Omega)$ are denoted $S^{1, p}(\Omega)$ and $\mathcal{L}^{1, p}(\Omega)$ respectively. In fact, it is only in Proposition A.4 where the argument in [4 must be essentially changed to accommodate our assumptions, namely that $\mathcal{X} \in H^{1, D}(\Omega)$ and that (38) holds with $\frac{1}{q}=\frac{1}{p}-\frac{1}{D}$, in place of the assumption in [4] that $\mathcal{X}$ is Lipschitz. Indeed, Proposition .7 is applied with $f=w \in \mathcal{L}^{1, p}(\Omega)=H_{\mathcal{X}}^{1, p}(\Omega)$, and since by (38) we now have $f \in L_{l o c}^{q}(\Omega)$, the analogue of Proposition .7 given below will complete the argument in [4]. We will use the notation in [4]: $J$ is a smooth function compactly supported in the unit ball $B$ in $\mathbb{R}^{n}, J_{\varepsilon}(x)=\varepsilon^{-n} J\left(\varepsilon^{-1} x\right)$, and

$$
\widetilde{J}_{\varepsilon}^{j}(x)=\left[X_{j}, J_{\varepsilon}\right]=X_{j} J_{\varepsilon}-J_{\varepsilon} X_{j}
$$

is the commutator of $X_{j}$ and $J_{\varepsilon}$. Lemma .6 in [4 shows that

$$
\widetilde{J}_{\varepsilon}^{j} f(x)=\int_{B} f(x+\varepsilon h) K_{\varepsilon}^{j}(x, h) d h,
$$

where

$$
K_{\varepsilon}^{j}(x, h)=\frac{1}{\varepsilon} \sum_{k=1}^{n} \frac{\partial}{\partial h_{k}}\left\{\left[b_{k}^{j}(x+\varepsilon h)-b_{k}^{j}(x)\right] J(h)\right\}
$$

and $X_{j}=\sum_{k=1}^{n} b_{k}^{j}(x) \frac{\partial}{\partial x_{k}}$ for $1 \leq j \leq m$. Here is the required analogue of Proposition .7 in [4].

Proposition 31. Let $\mathcal{X} \in H^{1, D}(\Omega)$. Then for any $f \in L_{l o c}^{q}(\Omega)$ with $\frac{1}{q}=\frac{1}{p}-\frac{1}{D}$ and $\omega \Subset \Omega$ we have

$$
\lim _{\varepsilon \rightarrow 0}\left\|\widetilde{J}_{\varepsilon}^{j} f\right\|_{L^{p}(\omega)}=0, \quad 1 \leq j \leq m .
$$

Proof. To see (39), fix $j$ and let $\widetilde{J}_{\varepsilon}, K_{\varepsilon}$ and $b_{k}$ denote $\widetilde{J}_{\varepsilon}^{j}, K_{\varepsilon}^{j}$ and $b_{k}^{j}$. By hypothesis $b_{k} \in H^{1, D}(\Omega)$. We have

$$
\left\|\widetilde{J}_{\varepsilon}^{j} f\right\|_{L^{p}(\omega)}=\sup _{\|g\|_{L^{p^{\prime}}(\omega)}=1}\left|\int_{\omega} \widetilde{J}_{\varepsilon}^{j} f(x) g(x) d x\right| .
$$

For $\|g\|_{L^{p^{\prime}(\omega)}}=1$ we compute using $\int_{B} K_{\varepsilon}(x, h) d h=0$, Hölder's inequality and $1=\frac{1}{q}+\frac{1}{D}+\frac{1}{p^{\prime}}$ that

$$
\begin{aligned}
\left|\int_{\omega} \widetilde{J}_{\varepsilon} f(x) g(x) d x\right| \\
=\left|\int_{\omega}\left\{\int_{B} f(x+\varepsilon h) K_{\varepsilon}(x, h) d h\right\} g(x) d x\right| \\
=\left|\int_{\omega}\left\{\int_{B}[f(x+\varepsilon h)-f(x)] K_{\varepsilon}(x, h) d h\right\} g(x) d x\right| \\
\quad \leq\left\{\int_{\omega} \int_{B}|f(x+\varepsilon h)-f(x)|^{q} d h d x\right\}^{\frac{1}{q}}\left\{\int_{\omega} \int_{B}\left|K_{\varepsilon}(x, h)\right|^{D} d h d x\right\}^{\frac{1}{D}},
\end{aligned}
$$


which tends to 0 as $\varepsilon \rightarrow 0$ since $f \in L_{l o c}^{q}(\Omega)$, translation is continuous in $L_{l o c}^{q}(\Omega)$, and

$$
\int_{\omega} \int_{B}\left|K_{\varepsilon}(x, h)\right|^{D} d h d x \leq C<\infty
$$

holds with $C$ independent of $\varepsilon<d(\partial \Omega, \omega)$. Indeed, to see (40) we write

$$
\begin{aligned}
K_{\varepsilon}(x, h) & =\sum_{k=1}^{n} \frac{\partial b_{k}}{\partial x_{k}}(x+\varepsilon h) J(h)+\frac{1}{\varepsilon} \sum_{k=1}^{n}\left[b_{k}(x+\varepsilon h)-b_{k}(x)\right] \frac{\partial J}{\partial h_{k}}(h) \\
& =I_{\varepsilon}(x, h)+I I_{\varepsilon}(x, h) .
\end{aligned}
$$

Now

$$
\left\{\int_{\omega} \int_{B}\left|I_{\varepsilon}(x, h)\right|^{D} d h d x\right\}^{\frac{1}{D}} \leq\left\|\sum_{k=1}^{n} \frac{\partial b_{k}}{\partial x_{k}}\right\|_{L^{D}(\Omega)}\|J\|_{L^{D}(B)}<\infty
$$

since $\varepsilon<d(\partial \Omega, \omega)$ and $b_{k} \in H^{1, D}(\Omega)$. Using

$$
b_{k}(x+\varepsilon h)-b_{k}(x)=\int_{0}^{1} \varepsilon h \cdot \nabla b_{k}(x+\varepsilon h t) d t,
$$

we also have

$$
\begin{aligned}
& \left\{\int_{\omega} \int_{B}\left|I I_{\varepsilon}(x, h)\right|^{D} d h d x\right\}^{\frac{1}{D}} \\
& \quad=\left\{\int_{\omega} \int_{B}\left|\sum_{k=1}^{n}\left[\int_{0}^{1} h \cdot \nabla b_{k}(x+\varepsilon h t) d t\right] \frac{\partial J}{\partial h_{k}}(h)\right|^{D} d h d x\right\}^{\frac{1}{D}} \\
& \leq \sum_{k=1}^{n}\left\{\int_{B}\left\{\int_{0}^{1} \int_{\omega}\left|\nabla b_{k}(x+\varepsilon h t)\right|^{D} d x d t\right\}\left|\frac{\partial J}{\partial h_{k}}(h) h\right|^{D} d h\right\}^{\frac{1}{D}} \\
& \leq \sum_{k=1}^{n}\left\|\nabla b_{k}\right\|_{L^{D}(\Omega)}\left\|\frac{\partial J}{\partial h_{k}}(h) h\right\|_{L^{D}(B)}<\infty .
\end{aligned}
$$

This completes the proof of (40) and hence of the proposition.

Now we can show that $J_{\varepsilon} * f \rightarrow f$ locally in $H_{\mathcal{X}}^{1, p}(\Omega)$ as $\varepsilon \rightarrow 0$ for every $f \in H_{\mathcal{X}}^{1, p}(\Omega)$. Indeed,

$$
\begin{aligned}
\left\|X_{j}\left(J_{\varepsilon} f\right)-X_{j} f\right\|_{L^{p}(\omega)} & =\left\|\left(J_{\varepsilon} X_{j}+\widetilde{J}_{\varepsilon}^{j}\right) f-X_{j} f\right\|_{L^{p}(\omega)} \\
& \leq\left\|J_{\varepsilon}\left(X_{j} f\right)-X_{j} f\right\|_{L^{p}(\omega)}+\left\|\widetilde{J}_{\varepsilon}^{j} f\right\|_{L^{p}(\omega)}
\end{aligned}
$$

tends to 0 as $\varepsilon \rightarrow 0$ for each $1 \leq j \leq m$, and obviously $\left\|J_{\varepsilon} f-f\right\|_{L^{p}(\omega)} \rightarrow 0$ as $\varepsilon \rightarrow 0$. 
With this accomplished we can now obtain the global result $W_{\mathcal{X}}^{1, p}(\Omega)=H_{\mathcal{X}}^{1, p}(\Omega)$ using a partition of unity argument as in [4].

5.2. Comparably Lipschitz off the common zero set. Now we turn to our second generalization of the $H=W$ theorem in 3] and [4. This time we assume that the vector fields $\mathcal{X}=\left\{X_{1}, \ldots, X_{m}\right\}$ are merely in $H^{1,2}(\Omega)$, but are comparably Lipschitz off their common zero set $Z$ : for each open set $\omega \Subset \Omega \backslash Z$ there is a positive constant $c_{\omega}$ and a collection of Lipschitz vector fields $\mathcal{F}_{\omega}=\left\{F_{1}, \ldots, F_{p}\right\}$, $p=p(\omega)$, defined in $\omega$ such that

$$
c_{\omega} \mathcal{F}_{\omega}(x, \xi) \leq \mathcal{X}(x, \xi) \leq \frac{1}{c_{\omega}} \mathcal{F}_{\omega}(x, \xi), \quad \xi \in \mathbb{R}^{n}, x \in \omega \Subset \Omega \backslash Z,
$$

where $Z=\bigcap_{j=1}^{m} Z_{j}$ and $Z_{j}=\left\{x \in \Omega: \mathbf{v}_{j}(x)=0\right\}$ is the zero set of the vector field $X_{j}(x)=\mathbf{v}_{j}(x) \cdot \nabla$. We prove that $H_{\mathcal{X}}^{1,2}=W_{\mathcal{X}}^{1,2}$ provided that in addition the vector fields $\mathcal{X}$ are "Lipschitz at their common zero set", i.e.

$$
\left|\mathbf{v}_{j}(x)\right| \leq \operatorname{Cdist}(x, Z), \quad x \in \Omega, 1 \leq j \leq m .
$$

Note in particular that (41) holds with $\mathcal{F}_{\omega}=\left\{\frac{\partial}{\partial x_{j}}\right\}_{j=1}^{n}$ if the vector fields $\mathcal{X} \in$ $H_{\text {div }}^{1,2}(\Omega)$ are elliptic off their common zero set $Z$ : for each compact subset $L$ of $\Omega \backslash Z$ there is a positive constant $c_{L}$ such that

$$
c_{L}|\xi|^{2} \leq \mathcal{X}(x, \xi)=\sum_{j=1}^{m}\left(\mathbf{v}_{j}(x) \cdot \xi\right)^{2} \leq \frac{1}{c_{L}}|\xi|^{2}, \quad \xi \in \mathbb{R}^{n}, x \in L .
$$

We also mention a weakening of the hypothesis (41), namely that the vector fields $\mathcal{F}_{\omega}$ need not be Lipschitz in $\omega$, but merely satisfy the hypotheses of Proposition 29 in $\omega$, i.e. $\mathcal{F}_{\omega} \in H^{1,2 \sigma^{\prime}}(\omega)$ and (16) holds with the same $\sigma$ for all compactly supported open subsets of $\omega$. Finally, in the subsection on examples below, we give an example of a collection $\mathcal{Y}=\left\{Y_{1}, Y_{2}, Y_{3}\right\}$ of three $H^{1,2}(\mathbb{D})$ vector fields in the unit disk $\mathbb{D}$ satisfying (43) and (42), but for which there is no collection $\mathcal{X}=\left\{X_{1}, \ldots, X_{m}\right\}$ of Lipschitz vector fields whose quadratic form $\mathcal{X}(x, \xi)$ is comparable to $\mathcal{Y}(x, \xi)$, thus demonstrating that Theorem 32 isn't a consequence of the Lipschitz result in [3] and 4 .

Theorem 32. Suppose that $\mathcal{X}=\left\{X_{1}, \ldots, X_{m}\right\}$ is an $H^{1,2}(\Omega)$ collection of vector fields in $\Omega \subset \mathbb{R}^{n}$ such that $\mathcal{X}$ is comparably Lipschitz off its common zero set and satisfies (42). Then $H_{\mathcal{X}}^{1,2}(\Omega)=W_{\mathcal{X}}^{1,2}(\Omega)$.

Proof. Let $Z=\bigcap_{j=1}^{m} Z_{j}$, where $Z_{j}$ is the zero set of the vector field $X_{j}=\mathbf{v}_{j} \cdot \nabla$. For $\delta \geq 0$ define the sets $P_{\delta}$ by

$$
P_{\delta}=\{x \in \Omega: \operatorname{dist}(x, Z)>\delta\} .
$$

We can construct smooth functions $\eta_{\delta}$ with $0 \leq \eta_{\delta} \leq 1$, and a positive constant $C$ such that

$$
\begin{aligned}
\eta_{\delta} & =1 \text { on } P_{2 \delta}, \\
\operatorname{supp}\left(\eta_{\delta}\right) & \subset P_{\delta}, \\
\left|\nabla \eta_{\delta}\right| & \leq C \delta^{-1} .
\end{aligned}
$$


Let $J$ be a smooth nonnegative function of integral one supported in the unit ball $B$ of $\mathbb{R}^{n}$, and as usual define

$$
J_{\varepsilon}(x)=\varepsilon^{-n} J\left(\varepsilon^{-1} x\right), \quad x \in \mathbb{R}^{n}, \varepsilon>0 .
$$

Let $f \in H_{\mathcal{X}}^{1,2}(\Omega)$ and $\delta>0$. Write

$$
f=g_{\delta}+b+h_{\delta}
$$

where

$$
\begin{aligned}
g_{\delta} & =\eta_{\delta} f, \\
b & =\chi_{Z} f, \\
h_{\delta} & =\chi_{P_{0}}\left(1-\eta_{\delta}\right) f .
\end{aligned}
$$

We will prove the following three assertions:

(1) $X_{j}\left(g_{\delta} * J_{\varepsilon}\right) \rightarrow X_{j} g_{\delta}$ in $L^{2}(K)$ as $\varepsilon \rightarrow 0$ for each compact set $K \subset \Omega$, $0<\delta<\operatorname{dist}\left(K, \Omega^{c}\right), 1 \leq j \leq m$, and

(2) $X_{j}\left(\left[\chi_{Z}\left(b * J_{\sigma}\right)\right] * J_{\varepsilon}\right) \rightarrow 0$ in $L^{2}(K)$ as $\varepsilon \rightarrow 0$ for each compact set $K \subset \Omega$, $0<\sigma<\frac{1}{2} \operatorname{dist}\left(K, \Omega^{c}\right), 1 \leq j \leq m$, and

(3) $\left\|h_{\delta}\right\|_{L^{2}(\Omega)}+\left\|X_{j} h_{\delta}\right\|_{L^{2}(\Omega)} \rightarrow 0$ as $\delta \rightarrow 0$ for each $1 \leq j \leq m$.

First we complete the demonstration of the theorem assuming these three assertions. In fact we now claim

$$
\limsup _{\varepsilon \rightarrow 0}\left\|f-\left(g_{\delta}+\chi_{Z}\left(b * J_{\sigma}\right)\right) * J_{\varepsilon}\right\|_{H_{\mathcal{X}}^{1,2}(\omega)} \rightarrow 0 \text { as } \sigma \text { and } \delta \rightarrow 0,
$$

for all open sets $\omega \Subset \Omega$. With $\operatorname{Lip}_{\mathcal{X}}(\omega)$ as defined in (13), we have $\left(g_{\delta}+\chi_{Z}\left(b * J_{\sigma}\right)\right)$ $* J_{\varepsilon} \in \operatorname{Lip} \mathcal{X}(\omega)$. It now follows easily that $H_{\mathcal{X}, l o c}^{1,2}(\Omega)=W_{\mathcal{X}, l o c}^{1,2}(\Omega)$ (as usual $B_{l o c}(\Omega)$ denotes those functions $f$ on $\Omega$ such that $\varphi f \in B(\Omega)$ for all $\varphi \in C_{c}^{\infty}(\Omega)$ ). A standard partition of unity argument $\left(\left[\underline{6}\right.\right.$; see also [4] ) now shows that $H_{\mathcal{X}}^{1,2}(\Omega)=$ $W_{\mathcal{X}}^{1,2}(\Omega)$. To see (44) we set $b_{\sigma}=\chi_{Z}\left(b * J_{\sigma}\right)$ on $\omega$ and use

$$
\begin{aligned}
f-\left(g_{\delta}+b_{\sigma}\right) * J_{\varepsilon} & =g_{\delta}+b+h_{\delta}-\left(g_{\delta}+b_{\sigma}\right) * J_{\varepsilon} \\
& =\left\{\left(g_{\delta}+b_{\sigma}\right)-\left(g_{\delta}+b_{\sigma}\right) * J_{\varepsilon}\right\}+h_{\delta}+b-b_{\sigma}
\end{aligned}
$$

to compute that

$$
\begin{aligned}
\left\|f-\left(g_{\delta}+b_{\sigma}\right) * J_{\varepsilon}\right\|_{L^{2}(\omega)} & \leq\left\|\left(g_{\delta}+b_{\sigma}\right)-\left(g_{\delta}+b_{\sigma}\right) * J_{\varepsilon}\right\|_{L^{2}(\omega)} \\
& +\left\|h_{\delta}\right\|_{L^{2}(\Omega)}+\left\|b-b_{\sigma}\right\|_{L^{2}(Z \cap \omega)},
\end{aligned}
$$

and since $g_{\delta}+b_{\sigma} \in L^{2}\left(\omega^{\prime}\right)$ with $\omega \Subset \omega^{\prime} \Subset \Omega$, we obtain

$$
\limsup _{\varepsilon \rightarrow 0}\left\|f-\left(g_{\delta}+b_{\sigma}\right) * J_{\varepsilon}\right\|_{L^{2}(\omega)} \leq\left\|h_{\delta}\right\|_{L^{2}(\Omega)}+\left\|b-b_{\sigma}\right\|_{L^{2}(Z \cap \omega)} .
$$

To estimate the $L^{2}$-norm of the derivatives we note that

$$
X_{j} f=X_{j}\left(g_{\delta}+h_{\delta}\right)
$$

in the weak sense in $\Omega$ since

$$
\begin{aligned}
\int \varphi X_{j} f=-\int f\left\{\nabla \cdot\left(\varphi \mathbf{v}_{j}\right)\right\} & =-\int \chi_{P_{0}} f\left\{\nabla \cdot\left(\varphi \mathbf{v}_{j}\right)\right\} \\
& =-\int\left(g_{\delta}+h_{\delta}\right)\left\{\nabla \cdot\left(\varphi \mathbf{v}_{j}\right)\right\}
\end{aligned}
$$


for $\varphi \in C_{c}^{1}(\Omega)$, where the first equality is the definition of $X_{j} f$, and the second equality follows from $P_{0}=\Omega \backslash Z$, the identity

$$
\nabla \cdot\left(\varphi \mathbf{v}_{j}\right)=\varphi \operatorname{div} \mathbf{v}_{j}+\nabla \varphi \cdot \mathbf{v}_{j}
$$

in Lemma 1, and the fact that $\operatorname{div} \mathbf{v}_{j}=0$ a.e. in the set $Z_{j}$ where $\mathbf{v}_{j}$ vanishes, which in turn holds since $\mathbf{v}_{j} \in H^{1,2}(\Omega)$ (see Lemma 7.7 in [5]). The third equality uses $\nabla \cdot\left(\varphi \mathbf{v}_{j}\right)=0$ a.e. in $Z$. Thus we compute

$$
\begin{aligned}
\left\|X_{j}\left\{f-\left(g_{\delta}+b_{\sigma}\right) * J_{\varepsilon}\right\}\right\|_{L^{2}(\omega)} & =\left\|X_{j}\left\{\left(g_{\delta}+h_{\delta}\right)-\left(g_{\delta}+b_{\sigma}\right) * J_{\varepsilon}\right\}\right\|_{L^{2}(\omega)} \\
& \leq\left\|X_{j}\left\{g_{\delta}-g_{\delta} * J_{\varepsilon}\right\}\right\|_{L^{2}(\omega)}+\left\|X_{j} h_{\delta}\right\|_{L^{2}(\Omega)} \\
& +\left\|X_{j}\left\{b_{\sigma} * J_{\varepsilon}\right\}\right\|_{L^{2}(\omega)}
\end{aligned}
$$

Now the first and third terms on the right side of (477) tend to 0 as $\varepsilon \rightarrow 0$ by assertions 1 and 2 above. Thus we have

$$
\begin{aligned}
\limsup _{\varepsilon \rightarrow 0}\left\|f-\left(g_{\delta}+b_{\sigma}\right) * J_{\varepsilon}\right\|_{H_{\mathcal{X}}^{1,2}(\omega)} \leq & \limsup _{\varepsilon \rightarrow 0}\left\|f-\left(g_{\delta}+b_{\sigma}\right) * J_{\varepsilon}\right\|_{L^{2}(\omega)} \\
& +\limsup _{\varepsilon \rightarrow 0}\left\|X_{j}\left\{f-\left(g_{\delta}+b_{\sigma}\right) * J_{\varepsilon}\right\}\right\|_{L^{2}(\omega)} \\
\leq \| & h_{\delta}\left\|_{L^{2}(\Omega)}+\right\| b-b * J_{\sigma} \|_{L^{2}(Z \cap \omega)} \\
& +\left\|X_{j} h_{\delta}\right\|_{L^{2}(\Omega)}
\end{aligned}
$$

The right-hand side of (48) tends to 0 as $\sigma$ and $\delta \rightarrow 0$ upon using $b \in L^{2}(\Omega)$ and assertion 3. This completes the proof of (44).

So it remains to prove assertions 1,2 and 3. Choose $K \subset \omega^{\prime} \Subset \Omega$. We obtain assertion 1 immediately from the final statement of Proposition 29 applied to the open set $\Omega \backslash Z$ together with assumption (41). Assertion 2 follows from the inequality $\left|\mathbf{v}_{j}\right| \leq C \varepsilon$ on $P_{0} \backslash P_{\varepsilon}$, which is in turn a consequence of (42). Indeed, using this with the estimate

$$
\left|\left[\nabla J_{\varepsilon}\right] *\left[\chi_{Z}\left(b * J_{\sigma}\right)\right](x)\right| \leq\left\|\nabla J_{\varepsilon}\right\|_{L^{1}}\left\|b * J_{\sigma}\right\|_{L^{\infty}\left(Z \cap \omega^{\prime}\right)} \leq C \frac{1}{\varepsilon}\left\|b * J_{\sigma}\right\|_{L^{\infty}\left(Z \cap \omega^{\prime}\right)}
$$

we have, since the support of $\left[\chi_{Z}\left(b * J_{\sigma}\right)\right] * J_{\varepsilon}$ is outside $P_{\varepsilon}$,

$$
\begin{aligned}
\int_{K}\left|X_{j}\left(\left[\chi_{Z}\left(b * J_{\sigma}\right)\right] * J_{\varepsilon}\right)\right|^{2} & =\int_{K \cap P_{0}}\left|\mathbf{v}_{j} \cdot \nabla\left(\left[\chi_{Z}\left(b * J_{\sigma}\right)\right] * J_{\varepsilon}\right)\right|^{2} \\
& =\int_{K \cap\left(P_{0} \backslash P_{\varepsilon}\right)}\left|\mathbf{v}_{j} \cdot\left(\left[\nabla J_{\varepsilon}\right] *\left[\chi_{Z}\left(b * J_{\sigma}\right)\right]\right)\right|^{2} \\
& \leq \int_{K \cap\left(P_{0} \backslash P_{\varepsilon}\right)}\left|\mathbf{v}_{j}(x)\right|^{2} \frac{C^{2}}{\varepsilon^{2}}\left\|b * J_{\sigma}\right\|_{L^{\infty}\left(Z \cap \omega^{\prime}\right)}^{2} d x \\
& \leq C^{2}\left\|b * J_{\sigma}\right\|_{L^{\infty}\left(Z \cap \omega^{\prime}\right)}^{2}\left|P_{0} \backslash P_{\varepsilon}\right| .
\end{aligned}
$$


The quantity $\left|P_{0} \backslash P_{\varepsilon}\right|$ tends to 0 as $\varepsilon \rightarrow 0$ since $\bigcap_{\varepsilon>0}\left(P_{0} \backslash P_{\varepsilon}\right)=\varnothing$, and this establishes assertion 2. Since $X_{j} b=0$ in the weak sense by (46), we have

$$
X_{j} h_{\delta}=X_{j}\left(b+h_{\delta}\right)=X_{j}\left(f-g_{\delta}\right)=X_{j}\left\{\left(1-\eta_{\delta}\right) f\right\} .
$$

Also (42) and supp $\nabla \eta_{\delta} \subset P_{\delta} \backslash P_{2 \delta}$ imply $\left|\mathbf{v}_{j}\right|\left|\nabla \eta_{\delta}\right| \leq C$. Assertion 3 now follows from

$$
\begin{aligned}
\int_{\Omega}\left|h_{\delta}\right|^{2}+\int_{\Omega}\left|X_{j} h_{\delta}\right|^{2}= & \int_{P_{0}}\left|\left(1-\eta_{\delta}\right) f\right|^{2}+\int_{\Omega}\left|\mathbf{v}_{j} \cdot \nabla\left\{\left(1-\eta_{\delta}\right) f\right\}\right|^{2} \\
\leq & C \int_{P_{0}}\left\{\left(1-\eta_{\delta}\right)^{2}+\left|\mathbf{v}_{j}\right|^{2}\left|\nabla \eta_{\delta}\right|^{2}\right\}|f|^{2} \\
& +C \int_{P_{0}}\left|\left(1-\eta_{\delta}\right)\right|^{2}\left|\mathbf{v}_{j} \cdot \nabla f\right|^{2} \\
\leq & C \int_{P_{0} \backslash P_{2 \delta}}\left(|f|^{2}+\left|X_{j} f\right|^{2}\right)
\end{aligned}
$$

which tends to 0 as $\delta \rightarrow 0$ since $\bigcap_{\delta>0}\left(P_{0} \backslash P_{2 \delta}\right)=\varnothing$.

5.3. One dimension. When $n=1$ and the vector fields $\mathcal{X}=\left\{X_{1}, \ldots, X_{m}\right\}$ are in $H^{1,2}(\Omega)$ (which coincides with $H_{\text {div }}^{1,2}(\Omega)$ in one dimension), $\Omega \subset \mathbb{R}$, we always have $H_{\mathcal{X}}^{1,2}(\Omega)=W_{\mathcal{X}}^{1,2}(\Omega)$.

Theorem 33. $H_{\mathcal{X}}^{1,2}(\Omega)=W_{\mathcal{X}}^{1,2}(\Omega)$ if $\mathcal{X}=\left\{X_{1}, \ldots, X_{m}\right\} \in H^{1,2}(\Omega), \Omega \subset \mathbb{R}$.

Proof. We suppose for convenience that there is only one vector field $X=v \frac{d}{d x}$, and that the open set $\Omega$ is a bounded interval. The proof in the general case is similar. The crucial consequence of $X \in H^{1,2}(\Omega)$ that is used in the approximation scheme is the lip $\frac{1}{2}$ estimate

$$
|v(y)-v(x)|=\left|\int_{x}^{y} v^{\prime}(t) d t\right| \leq\left(\int_{x}^{y}\left|v^{\prime}(t)\right|^{2} d t\right)^{\frac{1}{2}}|x-y|^{\frac{1}{2}} .
$$

Let $Z=\{x \in \Omega: v(x)=0\}$ and write

$$
\Omega \backslash Z=\bigcup_{n=1}^{\infty}=\bigcup_{n=1}^{\infty}\left(a_{n}, b_{n}\right)
$$

where $\bigcup$ denotes a pairwise disjoint union. We also define $P_{\delta}=\{x \in \Omega$ : $\operatorname{dist}(x, Z)>\delta\}$ for $\delta \geq 0$ as in the previous subsubsection. Note that $P_{0}=\Omega \backslash Z$.

Now suppose $f \in H_{\mathcal{X}}^{1,2}(\Omega)$ and $\varepsilon>0$ are given. We will complete the proof by constructing $g \in \operatorname{Lip}(\Omega)$ such that

$$
\|f-g\|_{H_{\mathcal{X}}^{1,2}(\Omega)}^{2}=\|f-g\|_{L^{2}(\Omega)}^{2}+\left\|f^{\prime}-g^{\prime}\right\|_{L^{2}\left(\Omega, v^{2}\right)}^{2}<C \varepsilon .
$$

First we choose $\varphi \in C_{c}^{\infty}(\Omega)$ such that

$$
\|f-\varphi\|_{L^{2}(\Omega)}^{2}<\varepsilon .
$$


Then since $\bigcap_{\delta>0}\left(P_{0} \backslash P_{\delta}\right)=\varnothing$, we can choose $\delta>0$ so that

$$
\begin{gathered}
\int_{P_{0} \backslash P_{\delta}}|f(x)|^{2} d x<\varepsilon \\
\int_{\left(P_{\delta}\right)^{c}}\left|f^{\prime}(x)\right|^{2} v(x)^{2} d x=\int_{P_{0} \backslash P_{\delta}}|X f(x)|^{2} d x<\varepsilon, \\
\int_{\left(P_{\delta}\right)^{c}}\left|\varphi^{\prime}(x)\right|^{2} v(x)^{2} d x=\int_{P_{0} \backslash P_{\delta}}|X \varphi(x)|^{2} d x<\varepsilon .
\end{gathered}
$$

Now since $\lim _{n \rightarrow \infty}\left|I_{n}\right|=0$, there are only finitely many of the intervals $I_{n}$ that intersect $P_{\delta}$ : enumerate them as $\left\{I_{n}\right\}_{n=1}^{N}$ and set $G=\bigcup_{n=1}^{N} I_{n}$. Note that $P_{\delta} \subset G$.

We now define $g$ in the set $\Omega \backslash G$ by $g=\varphi$. To define $g$ in the interval $I_{n}=\left(a_{n}, b_{n}\right)$ we proceed as follows. Suppose that both of the endpoints $a_{n}$ and $b_{n}$ lie in $Z$ (the case that one or both lie in $\partial \Omega$ is similar). Since $\left(a_{n}, b_{n}\right)$ intersects $P_{\delta}$ and $a_{n}, b_{n} \in Z$ we thus have $a_{n}<a_{n}+\delta<b_{n}-\delta<b_{n}$. Since $v$ is positive on $\left(a_{n}, b_{n}\right)$, $f$ is continuous on $\left(a_{n}, b_{n}\right)$ and we can choose a point $x_{n} \in\left(a_{n}+\frac{\delta}{2}, a_{n}+\delta\right)$ such that

$$
f\left(x_{n}\right)=\frac{2}{\delta} \int_{a_{n}+\frac{\delta}{2}}^{a_{n}+\delta} f(t) d t,
$$

and similarly choose $y_{n} \in\left(b_{n}-\delta, b_{n}-\frac{\delta}{2}\right)$ such that

$$
f\left(y_{n}\right)=\frac{2}{\delta} \int_{b_{n}-\delta}^{b_{n}-\frac{\delta}{2}} f(t) d t .
$$

Now define

$$
h_{n}(t)=\left\{\begin{array}{ccc}
f\left(x_{n}\right) & \text { for } & a_{n}<t \leq x_{n}, \\
f(t) & \text { for } & x_{n}<t<y_{n}, \\
f\left(y_{n}\right) & \text { for } & y_{n} \leq t<b_{n} .
\end{array}\right.
$$

Clearly $h_{n} \in L^{2}\left(I_{n}\right)$. We also have $h_{n}^{\prime}=f^{\prime} \chi_{\left(x_{n}, y_{n}\right)} \in L^{2}\left(I_{n}\right)$ since $f^{\prime} \in L^{2}\left(\Omega, v^{2}\right)$ and $v$ is positive on $\left(x_{n}, y_{n}\right)$, the support of $h_{n}^{\prime}$. Thus with $J$ as in the previous subsubsection, we can choose $0<\sigma<\frac{\delta}{4}$ independent of $1 \leq n \leq N$ so small that (for the purpose of defining the convolution $h_{n} * J_{\sigma}$ we extend $h_{n}$ to be continuous and constant outside $I_{n}$ )

$$
\begin{aligned}
\left\|h_{n} * J_{\sigma}-h_{n}\right\|_{L^{2}\left(I_{n}\right)}^{2}<\frac{\varepsilon}{N}, \\
\left\|h_{n}^{\prime} * J_{\sigma}-h_{n}^{\prime}\right\|_{L^{2}\left(I_{n}\right)}^{2}<\frac{\varepsilon}{N} .
\end{aligned}
$$

The function $h_{n} * J_{\sigma}$ is smooth in $I_{n}$ and since $\sigma<\frac{\delta}{4}$, it is constant on the intervals $\left(a_{n}, a_{n}+\frac{\delta}{4}\right)$ and $\left(b_{n}-\frac{\delta}{4}, b_{n}\right)$, taking the values $f\left(x_{n}\right)$ and $f\left(y_{n}\right)$ there respectively. The only reason we cannot define $g$ to be $h_{n} * J_{\sigma}$ on $I_{n}$ is that $h_{n} * J_{\sigma}$ does not match $\varphi$ at the endpoints $a_{n}$ and $b_{n}$. So we will modify $h_{n} * J_{\sigma}$ near the endpoints to match $\varphi$ there by adding a small piecewise affine perturbation $\psi_{n}$, and use the lip $\frac{1}{2}$ estimate (49) on $v$ to control the $L^{2}\left(v^{2}\right)$-norm of the derivative of the perturbation. Here are the details. For $0<\tau<\frac{\delta}{4}$ to be chosen momentarily, we define $\psi_{n}$ to be the piecewise affine function on $\left[a_{n}, b_{n}\right]$ whose graph joins the four points $\left(a_{n}, \varphi\left(a_{n}\right)-f\left(x_{n}\right)\right),\left(a_{n}+\tau, 0\right),\left(b_{n}-\tau, 0\right)$ and $\left(b_{n}, \varphi\left(b_{n}\right)-f\left(y_{n}\right)\right)$ in 
succession. Using (49) and $a_{n}, b_{n} \in Z$ we have for $1 \leq n \leq N$,

$$
\begin{aligned}
\int_{I_{n}}\left|\psi_{n}^{\prime}\right|^{2} v^{2} & =\int_{a_{n}}^{a_{n}+\tau} \frac{\left|\varphi\left(a_{n}\right)-f\left(x_{n}\right)\right|^{2}}{\tau^{2}} v(t)^{2} d t \\
& +\int_{b_{n}-\tau}^{b_{n}} \frac{\left|\varphi\left(b_{n}\right)-f\left(y_{n}\right)\right|^{2}}{\tau^{2}} v(t)^{2} d t \\
& \leq \frac{\left|\varphi\left(a_{n}\right)-f\left(x_{n}\right)\right|^{2}}{\tau^{2}}\left(\int_{a_{n}}^{a_{n}+\tau}\left|v^{\prime}\right|^{2}\right) \tau \int_{a_{n}}^{a_{n}+\tau} d t \\
& +\frac{\left|\varphi\left(b_{n}\right)-f\left(y_{n}\right)\right|^{2}}{\tau^{2}}\left(\int_{b_{n}-\tau}^{b_{n}}\left|v^{\prime}\right|^{2}\right) \tau \int_{b_{n}-\tau}^{b_{n}} d t \\
& \leq\left|\varphi\left(a_{n}\right)-f\left(x_{n}\right)\right|^{2} \int_{a_{n}}^{a_{n}+\tau}\left|v^{\prime}\right|^{2}+\left|\varphi\left(b_{n}\right)-f\left(y_{n}\right)\right|^{2} \int_{b_{n}-\tau}^{b_{n}}\left|v^{\prime}\right|^{2}<\frac{\varepsilon}{N}
\end{aligned}
$$

if $\tau>0$ is chosen sufficiently small. We also choose $\tau>0$ small enough to ensure

$$
\left\{\left|\varphi\left(a_{n}\right)-f\left(x_{n}\right)\right|^{2}+\left|\varphi\left(b_{n}\right)-f\left(y_{n}\right)\right|^{2}\right\} \tau<\frac{\varepsilon}{N}, \quad 1 \leq n \leq N .
$$

Then we set $g=h_{n} * J_{\sigma}+\psi_{n}$ on $I_{n}$, for $1 \leq n \leq N$. Altogether we have

$$
g(t)=\left\{\begin{array}{cl}
\varphi(t) & \text { for } \quad t \in \Omega \backslash G, \\
h_{n} * J_{\sigma}(t)+\psi_{n}(t) & \text { for } \quad t \in I_{n}, \quad 1 \leq n \leq N .
\end{array}\right.
$$

Clearly $g \in \operatorname{Lip}(\Omega)$ and we have

$$
f-g=(f-\varphi) \chi_{\Omega \backslash G}+\sum_{n=1}^{N}\left(f-h_{n}\right) \chi_{I_{n}}+\sum_{n=1}^{N}\left(h_{n}-h_{n} * J_{\sigma}\right) \chi_{I_{n}}-\sum_{n=1}^{N} \psi_{n} .
$$

We now use (56) to compute $\|f-g\|_{L^{2}(\Omega)}$ and $\|X f-X g\|_{L^{2}(\Omega)}$ as follows:

$$
\begin{aligned}
\|f-g\|_{L^{2}(\Omega)}^{2} & \lesssim\|f-\varphi\|_{L^{2}(\Omega)}^{2}+\sum_{n=1}^{N} \int_{a_{n}}^{b_{n}}\left|f-h_{n}\right|^{2} \\
& +\sum_{n=1}^{N} \int_{a_{n}}^{b_{n}}\left|h_{n}-h_{n} * J_{\sigma}\right|^{2}+\sum_{n=1}^{N} \int_{a_{n}}^{b_{n}}\left|\psi_{n}\right|^{2} \\
& =I+I I+I I I+I V,
\end{aligned}
$$

where $I<\varepsilon$ by (51),

$$
\begin{aligned}
& I I \leq \sum_{n=1}^{N}\left\{\int_{a_{n}}^{x_{n}}\left|f-\frac{2}{\delta} \int_{a_{n}+\frac{\delta}{2}}^{a_{n}+\delta} f\right|^{2}+\int_{y_{n}}^{b_{n}}\left|f-\frac{2}{\delta} \int_{b_{n}-\delta}^{b_{n}-\frac{\delta}{2}} f\right|^{2}\right\} \\
& \leq C \sum_{n=1}^{N}\left\{\int_{a_{n}}^{x_{n}}|f|^{2}+\frac{2\left(x_{n}-a_{n}\right)}{\delta} \int_{a_{n}+\frac{\delta}{2}}^{a_{n}+\delta}|f|^{2}+\int_{y_{n}}^{b_{n}}|f|^{2}\right. \\
&\left.+\frac{2\left(b_{n}-y_{n}\right)}{\delta} \int_{b_{n}-\delta}^{b_{n}-\frac{\delta}{2}}|f|^{2}\right\} \\
& \leq C \int_{P_{0} \backslash P_{\delta}}|f|^{2}<C \varepsilon
\end{aligned}
$$


by the first line in (52), III $<\sum_{n=1}^{N} \frac{\varepsilon}{N}=\varepsilon$ by the first line in (153), and

$$
I V \leq \sum_{n=1}^{N}\left\{\left|\varphi\left(a_{n}\right)-f\left(x_{n}\right)\right|^{2}+\left|\varphi\left(b_{n}\right)-f\left(y_{n}\right)\right|^{2}\right\} \tau<\sum_{n=1}^{N} \frac{\varepsilon}{N}=\varepsilon
$$

by (55).

Using (56) again, together with $P_{\delta} \subset G$, the derivatives are estimated by

$$
\begin{aligned}
\|X f-X g\|_{L^{2}(\Omega)}^{2} & \leq \int_{\left(P_{\delta}\right)^{c}}|X f|^{2}+\int_{\left(P_{\delta}\right)^{c}}|X \varphi|^{2}+\sum_{n=1}^{N} \int_{a_{n}}^{b_{n}}\left|X f-X h_{n}\right|^{2} \\
& +\sum_{n=1}^{N} \int_{a_{n}}^{b_{n}}\left|X\left(h_{n}-h_{n} * J_{\sigma}\right)\right|^{2}+\sum_{n=1}^{N} \int_{a_{n}}^{b_{n}}\left|X \psi_{n}\right|^{2} \\
& =V+V I+V I I+V I I I+I X,
\end{aligned}
$$

where $V+V I<2 \varepsilon$ by the second two lines in (52),

$$
V I I=\sum_{n=1}^{N}\left\{\int_{a_{n}}^{x_{n}}|X f|^{2}+\int_{y_{n}}^{b_{n}}|X f|^{2}\right\} \leq \int_{\left(P_{\delta}\right)^{c}}|X f|^{2}<\varepsilon
$$

by the second line in (52),

$$
V I I I \leq C\|v\|_{L^{\infty}(\Omega)}^{2} \sum_{n=1}^{N} \int_{a_{n}}^{b_{n}}\left|h_{n}^{\prime}-h_{n}^{\prime} * J_{\sigma}\right|^{2} \leq C\|v\|_{L^{\infty}(\Omega)}^{2} \sum_{n=1}^{N} \frac{\varepsilon}{N}=C^{\prime} \varepsilon
$$

by the second line in (53), and finally $I X \leq \sum_{n=1}^{N} \frac{\varepsilon}{N}=\varepsilon$ by (54). This completes the proof of (50), and hence that of Theorem 33

Remark 34. Theorem 33 generalizes to show that $H_{\mathcal{X}}^{1, p}(\Omega)=W_{\mathcal{X}}^{1, p}(\Omega)$ for $1<p \leq 2$ provided $n=1$ and the vector fields $\mathcal{X}$ are in $H^{1, p^{\prime}}(\Omega)$. Here $H_{\mathcal{X}}^{1, p}(\Omega)$ and $W_{\mathcal{X}}^{1, p}(\Omega)$ are defined as in Remarks 3 and 9 above. In fact, only inequality (54) of the proof of Theorem 33 is affected for $p \neq 2$ and now becomes

$$
\begin{aligned}
\int_{I_{n}}\left|\psi_{n}^{\prime}\right|^{p} v^{p} & \leq \tau^{2-p}\left|\varphi\left(a_{n}\right)-f\left(x_{n}\right)\right|^{p}\left(\int_{a_{n}}^{a_{n}+\tau}\left|v^{\prime}\right|^{p^{\prime}}\right)^{p-1} \\
& +\tau^{2-p}\left|\varphi\left(b_{n}\right)-f\left(y_{n}\right)\right|^{p}\left(\int_{b_{n}-\tau}^{b_{n}}\left|v^{\prime}\right|^{p^{\prime}}\right)^{p-1} .
\end{aligned}
$$

For $1<p \leq 2$, the right side of (57) tends to 0 as $\tau \rightarrow 0$, and the proof of Theorem 33 shows that $H_{\mathcal{X}}^{1, p}(\Omega)=W_{\mathcal{X}}^{1, p}(\Omega)$. However, when $p>2$, the right side of (57) need not tend to 0 as $\tau \rightarrow 0$, resulting in examples for which $H_{\mathcal{X}}^{1, p}(\Omega) \neq W_{\mathcal{X}}^{1, p}(\Omega)$, even when $\mathcal{X} \in H^{1, q}(\Omega)$ for all $q<p$. See the subsection on examples below.

5.4. Examples. We first give an example of a collection $\mathcal{Y}=\left\{Y_{1}, Y_{2}, Y_{3}\right\}$ of three $H^{1,2}(\mathbb{D})$ vector fields in the unit disk $\mathbb{D}$ such that $\mathcal{Y}$ is elliptic off its common zero set and satisfies (42), but for which there is no collection $\mathcal{X}=\left\{X_{1}, \ldots, X_{m}\right\}$ of Lipschitz vector fields whose quadratic form $\mathcal{X}(x, \xi)$ is comparable to $\mathcal{Y}(x, \xi)$. This shows that Theorem 32 is not included in the known theorems in [3] and [4.

Example 35. Identify $x=\left(x_{1}, x_{2}\right) \in \mathbb{R}^{2}$ with $x=x_{1}+i x_{2}=r e^{i \theta} \in \mathbb{C}$, and define $\mathbb{C}$-valued functions $\mathbf{w}_{1}, \mathbf{w}_{2}, \mathbf{w}_{3}$ by

$$
\mathbf{w}_{1}(x)=e^{-r^{-2}}, \quad \mathbf{w}_{2}(x)=i e^{-r^{-2}}, \quad \mathbf{w}_{3}(x)=x e^{i r^{-\alpha}}=r e^{i\left(\theta+r^{-\alpha}\right)},
$$


so that $Y_{j}(x)=\mathbf{w}_{j}(x) \cdot \nabla$ is given by

$$
\begin{aligned}
& Y_{1}(x)=e^{-r^{-2}} \frac{\partial}{\partial x_{1}}, \\
& Y_{2}(x)=e^{-r^{-2}} \frac{\partial}{\partial x_{2}}, \\
& Y_{3}(x)=r \cos \left(\theta+r^{-\alpha}\right) \frac{\partial}{\partial x_{1}}+r \sin \left(\theta+r^{-\alpha}\right) \frac{\partial}{\partial x_{2}} .
\end{aligned}
$$

The vectors $\mathbf{w}_{1}$ and $\mathbf{w}_{2}$ are smooth, and $\mathbf{w}_{3} \in H^{1,2}(\mathbb{D})$ for $-\infty<\alpha<1$ since $\frac{\partial}{\partial r}\left(r \cos \left(\theta+r^{-\alpha}\right)\right)=O\left(1+r^{-\alpha}\right)$ and $\frac{1}{r} \frac{\partial}{\partial \theta}\left(r \cos \left(\theta+r^{-\alpha}\right)\right)=O(1)$, etc., but $\mathbf{w}_{3}$ is Lipschitz only for $\alpha \leq 0$ (and also with $r^{-\alpha}$ replaced by $\log r$ ). Conditions (43) and (42) hold with $Z=\{0\}$ for all $\alpha$.

However, if $\mathcal{X}=\left\{X_{1}, \ldots, X_{m}\right\}$ is a collection of vector fields $X_{j}=\mathbf{v}_{j} \cdot \nabla$ satisfying

$$
\left.\mathcal{X}(x, \xi) \approx \mathcal{Y}(x, \xi) \equiv \sum_{j=1}^{3}\left(\mathbf{w}_{j}(x) \cdot \xi\right)^{2}=e^{-2 r^{-2}}|\xi|^{2}+\left\{\operatorname{Re}\left(r e^{i\left(\theta+r^{-\alpha}\right.}\right) \bar{\xi}\right)\right\}^{2},
$$

then for $\xi=\mathbf{e}_{1}$ and $x=(r, 0)$, which is denoted $r \in \mathbb{C}$, we have

$$
\sum_{j=1}^{m}\left(\mathbf{v}_{j}(r) \cdot \mathbf{e}_{1}\right)^{2}=\mathcal{X}\left(r, \mathbf{e}_{1}\right) \approx e^{-2 r^{-2}}+r^{2} \cos ^{2}\left(r^{-\alpha}\right),
$$

which shows that $\mathcal{X}$ cannot be Lipschitz if $\alpha>0$. Indeed, if $\mathcal{X}$ is Lipschitz, then

$$
\begin{aligned}
\left|\mathcal{X}\left(r+s, \mathbf{e}_{1}\right)-\mathcal{X}\left(r, \mathbf{e}_{1}\right)\right| & =\left|\sum_{j=1}^{m}\left\{\left(\mathbf{v}_{j}(r+s) \cdot \mathbf{e}_{1}\right)^{2}-\left(\mathbf{v}_{j}(r) \cdot \mathbf{e}_{1}\right)^{2}\right\}\right| \\
& \leq C|s| \sum_{j=1}^{m}\left|\mathbf{v}_{j}(r+s) \cdot \mathbf{e}_{1}+\mathbf{v}_{j}(r) \cdot \mathbf{e}_{1}\right| \\
& \leq C|s|(r+|s|),
\end{aligned}
$$

since $\mathcal{X}\left(x, \mathbf{e}_{1}\right) \leq C \mathcal{Y}\left(x, \mathbf{e}_{1}\right) \leq C r^{2}$. On the other hand, if we choose $0<r_{0}<\frac{1}{10}$ so that $\cos \left(r_{0}^{-\alpha}\right)=0$, and then take $s_{0}>0$ such that $\left(r_{0}+s_{0}\right)^{-\alpha}=r_{0}^{-\alpha}-\frac{\pi}{2}$, we have

$$
\frac{\pi}{2} r_{0}^{\alpha}=1-\left(1+\frac{s_{0}}{r_{0}}\right)^{-\alpha} \approx 1-\left(1-\alpha \frac{s_{0}}{r_{0}}\right)=\alpha \frac{s_{0}}{r_{0}}
$$

and so

$$
s_{0} \approx \frac{\pi}{2 \alpha} r_{0}^{1+\alpha} .
$$

But (58) and $\cos \left(r_{0}^{-\alpha}\right)=0$ yield

$$
\begin{gathered}
\mathcal{X}\left(r_{0}, \mathbf{e}_{1}\right) \leq C e^{-2 r_{0}^{-2}}, \\
\mathcal{X}\left(r_{0}+s_{0}, \mathbf{e}_{1}\right) \geq c\left(r_{0}+s_{0}\right)^{2},
\end{gathered}
$$

and so we have

$$
\mathcal{X}\left(r_{0}+s_{0}, \mathbf{e}_{1}\right)-\mathcal{X}\left(r_{0}, \mathbf{e}_{1}\right) \geq c\left(r_{0}+s_{0}\right)^{2}-C e^{-2 r_{0}^{-2}} \approx r_{0}^{2} \gg r_{0}^{2+\alpha} \approx s_{0}\left(r_{0}+s_{0}\right),
$$

contradicting (59). 
We observe that the above argument yields a single vector field that is comparably Lipschitz, instead of elliptic, off its zero set:

Example 36. The single vector field $\mathcal{Y}=\left\{Y_{3}\right\}$ is comparably Lipschitz off its zero set and satisfies (42) in $\mathbb{D}$, while by the argument above, $\mathcal{Y}(x, \xi)$ fails to be comparable to $\mathcal{X}(x, \xi)$ for any collection $\mathcal{X}=\left\{X_{1}, \ldots, X_{m}\right\}$ of Lipschitz vector fields in $\mathbb{D}$.

Next we give an example of nonvanishing and non-Lipschitz vector fields that satisfy the hypotheses of Proposition 29.

Example 37. Let $\alpha$ and $\beta$ be positive rational fractions with even numerators and odd denominators, and suppose

$$
1>\alpha>\frac{\sqrt{5}-1}{2}, \quad 1>\beta>\frac{1+\alpha}{2+\alpha} .
$$

Set $D=2+\alpha$. We claim that the vector fields $\mathcal{X}=\{X, Y\}$, defined in the unit disk $\mathbb{D}$ by

$$
X=\frac{\partial}{\partial x}+y^{\beta} \frac{\partial}{\partial y} \text { and } Y=\frac{\partial}{\partial x}+x^{\alpha} \frac{\partial}{\partial y},
$$

are in $H^{1, D}(\mathbb{D})$ and satisfy (38) for $\frac{1}{q}=\frac{1}{p}-\frac{1}{D}$ (they are Lipschitz only for $\alpha, \beta \geq 1$ ). The integral curves for $X$ are given by

$$
\gamma(t)=(t, 0) \text { and } \gamma_{a}(t)=\left(t,[(1-\beta)(t-a)]^{\frac{1}{1-\beta}}\right),
$$

together with concatenations of $\gamma_{a_{1}}, \gamma$ and $\gamma_{a_{2}}$ that join on the $x$-axis. The integral curves for $Y$ are given by

$$
\delta_{b}(t)=\left(t, b+\frac{t^{1+\alpha}}{1+\alpha}\right) .
$$

Note that both of the exponents $\frac{1}{1-\beta}$ and $1+\alpha$ are fractions with odd denominator by our assumptions on $\alpha$ and $\beta$. The hypothesis $\beta>\frac{1+\alpha}{2+\alpha}$ implies that $1+\alpha<\frac{1}{1-\beta}$, and a long and tedious calculation using $\gamma_{a}$ and $\delta_{b}$ then shows that the control balls $B((x, y), r)$ associated with $\mathcal{X}$ have doubling constant $D=2+\alpha$, i.e.

$$
|B((x, y), r)| \leq C\left(\frac{r}{s}\right)^{2+\alpha}|B((x, y), s)|,
$$

for all $0<s \leq r \leq 1$ and $(x, y) \in \mathbb{D}$. Using the integral curves $\gamma_{a}$ and $\delta_{b}$ we can establish the subrepresentation inequality (186) in [11, and then Proposition 74 of 11 yields the Sobolev inequality (38) with $D=2+\alpha$. Now $\mathcal{X} \in H^{1, D}(\mathbb{D})$ provided $\alpha, \beta>1-\frac{1}{D}$, and this follows from $D=2+\alpha, \alpha>\frac{\sqrt{5}-1}{2}$ and $\beta>\frac{1+\alpha}{2+\alpha}$. Finally, arguing as in the first example, we can show that $\mathcal{X}(x, \xi)$ is not comparable to any quadratic form arising from Lipschitz vector fields.

Convolution of elements in $H_{\mathcal{X}}^{1,2}(\Omega)$ is not in general sufficient for proving density of $\operatorname{Lip}(\Omega)$ when $\mathcal{X}$ is not Lipschitz. Here is an example in dimension $n=1$.

Example 38. If

$$
v_{\beta}(x)=\left(x_{+}\right)^{\beta}=\left\{\begin{array}{ccc}
0 & \text { if } & -1<x \leq 0, \\
x^{\beta} & \text { if } \quad 0<x<1,
\end{array}\right.
$$

on $\Omega \equiv(-1,1)$, then $v_{\beta} \in H^{1,2}(\Omega)$ for all $\beta>\frac{1}{2}$, yet for $\mathcal{X}_{\beta}=v_{\beta} \frac{d}{d x}$, the convolutions $f * J_{\varepsilon} \rightarrow f$ in $H_{\mathcal{X}_{\beta}}^{1,2}(-1,1)$ for all $f \in H_{\mathcal{X}_{\beta}}^{1,2}(-1,1)$ if and only if $\beta \geq 1$. 
Indeed, $f_{\alpha}(x)=\left(x_{-}\right)^{-\alpha} \in H_{\mathcal{X}_{\beta}}^{1,2}(-1,1)$ provided $\alpha<\frac{1}{2}$ since $v_{\beta} \frac{d}{d x} f_{\alpha}=0$ in the weak sense. Assuming $J \geq 0$ one then computes that for $2 \varepsilon<x<3 \varepsilon$,

$$
\left(J_{\varepsilon} * f_{\alpha}\right)^{\prime}(x)=J_{\varepsilon} * f_{\alpha}^{\prime}(x)=\alpha \int_{-\varepsilon}^{\varepsilon}\left((x-t)_{-}\right)^{-\alpha-1} \varepsilon^{-1} J\left(\varepsilon^{-1} t\right) d t \geq c \varepsilon^{-\alpha-1},
$$

for $\alpha>0$, and hence

$$
\begin{aligned}
\left\|f_{\alpha}-J_{\varepsilon} * f_{\alpha}\right\|_{H_{\mathcal{X}_{\beta}}^{1,2}(\Omega)}^{2} & \geq\left\|f_{\alpha}^{\prime}-\left(J_{\varepsilon} * f_{\alpha}\right)^{\prime}\right\|_{L^{2}\left(\Omega, v_{\beta}^{2}\right)}^{2} \\
& \geq \int_{2 \varepsilon}^{3 \varepsilon}\left|\left(J_{\varepsilon} * f_{\alpha}\right)^{\prime}(x)\right|^{2} v_{\beta}(x)^{2} d x \\
& \geq\left(c \varepsilon^{-\alpha-1}\right)^{2} \int_{2 \varepsilon}^{3 \varepsilon} x^{2 \beta} d x=c \varepsilon^{2(\beta-\alpha)-1}
\end{aligned}
$$

tends to $\infty$ as $\varepsilon \rightarrow 0$ if $\alpha>\beta-\frac{1}{2}$. Thus given $\frac{1}{2}<\beta<1$, we can choose $\alpha>0$ satisfying $\beta-\frac{1}{2}<\alpha<\frac{1}{2}$ so that $f_{\alpha} \in H_{\mathcal{X}^{\beta}}^{1,2}(\Omega)$ and $J_{\varepsilon} * f_{\alpha} \nrightarrow f_{\alpha}$ locally in $H_{\mathcal{X}^{\beta}}^{1,2}(\Omega)$.

Now we give examples in all dimensions of vector fields $\mathcal{X}$ in $H^{1, p^{\prime}}(\Omega)$ for which $H_{\mathcal{X}}^{1, p}(\Omega) \neq W_{\mathcal{X}}^{1, p}(\Omega), p>2$; compare Remark 34

Example 39. For every $p>2$ and $n \geq 1$ there is a vector field $X \in H^{1, p^{\prime}}(\Omega)$ for which $H_{\mathcal{X}}^{1, p}(\Omega) \neq W_{\mathcal{X}}^{1, p}(\Omega), \mathcal{X}=\{X\}$, and moreover, we can even take $X \in$ $H^{1, q}(\Omega)$ for $p^{\prime} \leq q<p$. To see this in dimension $n=1$, fix $\frac{1}{p} \leq \frac{1}{q^{\prime}}<\beta<\frac{1}{p^{\prime}}$ and let $X=|x|^{\beta} \frac{d}{d x}$ for $x \in \Omega \equiv(-1,1)$. Then $X \in H^{1, q}(\Omega)$ since $\beta>\frac{1}{q^{\prime}}$. Set $u(x)=\operatorname{sgn}(x)$ so that $X u=0$ in the weak sense and $u \in H_{\mathcal{X}}^{1, p}(\Omega)$. Now we observe that if $w \in \operatorname{Lip}(\Omega) \cap H_{\mathcal{X}}^{1, p}(\Omega)=\operatorname{Lip}_{\mathcal{X}, p}(\Omega)$, then $\beta<\frac{1}{p^{\prime}}$ yields the Poincaré inequality:

$$
\begin{aligned}
\int_{\Omega}|w(x)-w(0)|^{p} d x & =\int_{-1}^{1}\left|\int_{0}^{x} w^{\prime}(t) d t\right|^{p} d x=\left.\left.\int_{-1}^{1}\left|\int_{0}^{x}\right| t\right|^{-\beta}(X w)(t) d t\right|^{p} d x \\
& \leq\left.\left.\left.\int_{-1}^{1}\left|\int_{0}^{x}\right| t\right|^{-\beta p^{\prime}} d t\right|^{p-1}\left|\int_{0}^{x}\right|(X w)(t)\right|^{p} d t \mid d x \\
& \leq\left.\left. C_{p, \beta} \int_{-1}^{1}\left|\int_{0}^{x}\right|(X w)(t)\right|^{p} d t\left|d x \leq C_{p, \beta} \int_{\Omega}\right|(X w)(t)\right|^{p} d t .
\end{aligned}
$$

From this and $X u=0$ we easily see that $u \notin W_{\mathcal{X}}^{1, p}(\Omega)$. Indeed, if $w \in \operatorname{Lip}_{\mathcal{X}, p}(\Omega)$, then

$$
\begin{aligned}
C_{p, \beta}^{\frac{1}{p}}\|X(w-u)\|_{L^{p}(\Omega)} & =C_{p, \beta}^{\frac{1}{p}}\|X w\|_{L^{p}(\Omega)} \\
& \geq\|w-w(0)\|_{L^{p}(\Omega)} \\
& \geq\|u-w(0)\|_{L^{p}(\Omega)}-\|w-u\|_{L^{p}(\Omega)} \\
& \geq 1-\|w-u\|_{L^{p}(\Omega)}
\end{aligned}
$$

shows that $\|w-u\|_{H_{\mathcal{X}}^{1, p}(\Omega)} \geq c_{p, \beta}>0$ for all $w \in \operatorname{Lip}_{\mathcal{X}, p}(\Omega)$; hence $u$ is not in the closure $W_{\mathcal{X}}^{1, p}(\Omega)$ of $\operatorname{Lip}_{\mathcal{X}, p}(\Omega)$ in $H_{\mathcal{X}}^{1, p}(\Omega)$. This example immediately lifts to higher dimensions by introducing extra variables and then multiplying the function $u$ by an appropriate smooth cutoff function. 
Remark 40. Note that the existence of a Poincaré inequality as above prohibits $\operatorname{sgn}(x)$ from belonging to $W_{\mathcal{X}}^{1, p}(\Omega)$. On the other hand, $\operatorname{sgn}(x)$ belongs to $H_{\mathcal{X}}^{1, p}(\Omega)$ if $X$ vanishes on the $y$-axis. The above example shows that both phenomena can occur simultaneously if $p>2$.

\section{REFERENCES}

[1] R. Bouldin, The norm continuity properties of square roots, SIAM J. Math. Anal. 4 (1972). MR0310673 (46:9771)

[2] E. Fabes, C. Kenig and R. Serapioni, The local regularity of solutions of degenerate elliptic equations, Comm. P.D.E. 7 (1982), 77-116. MR643158(84i:35070)

[3] B. Franchi, R. Serapioni and F. Serra Cassano, Meyers-Serrin type theorems and relaxation of variational integrals depending on vector fields, Houston J. Math. 22 (1996), 859-889. MR 1437714 (98c:49037)

[4] N. Garofalo And D. M. Nhieu, Isoperimetric and Sobolev inequalities for CarnotCarathéodory spaces and the existence of minimal surfaces, Comm. Pure Appl. Math. 49 (1996), 1081-1144. MR1404326 (97i:58032)

[5] D. Gilbarg and N. Trudinger, Elliptic Partial Differential Equations of Second Order, Springer-Verlag, Berlin, 2001. MR.1814364 (2001k:35004)

[6] N. Meyers and J. Serrin, $H=W$, Proc. Nat. Acad. Sci. U.S.A. 51 (1964), 1055-1056. MR0164252 (29:1551)

[7] C. Rios, E. Sawyer and R. L. Wheeden, A higher-dimensional partial Legendre transform, and regularity of degenerate Monge-Ampère equations, Advances in Mathematics 193 (2005), 373-415. MR2137289 (2006f:35099)

[8] C. Rios, E. SAWYer and R. L. Wheeden, Regularity of subelliptic Monge-Ampère equations, Advances in Mathematics 217 (2008), 967-1026. MR2383892

[9] C. Rios, E. Sawyer and R. L. Wheeden, Hypoellipticity for infinitely degenerate quasilinear equations and the Dirichlet problem, in preparation.

[10] S. Rodney, Existence of weak solutions to subelliptic partial differential equations in divergence form and the necessity of the Sobolev and Poincaré inequalities, thesis.

[11] E. SAwyer and R. L. Wheeden, Hölder continuity of subelliptic equations with rough coefficients, Memoirs Amer. Math. Soc. 847 (2006). MR2204824 (2007f:35037)

Department of Mathematics, McMaster University, Hamilton, Ontario, Canada L8S $4 \mathrm{~K} 1$

Department of Mathematics, Rutgers University, New Brunswick, New Jersey 08854 